\title{
COMPETITION IN THE CHEMOSTAT: A DISTRIBUTED DELAY MODEL AND ITS GLOBAL ASYMPTOTIC BEHAVIOR*
}

\author{
GAIL S. K. WOLKOWICZ ${ }^{\dagger}$, HUAXING XIA ${ }^{\dagger}$, AND SHIGUI RUAN ${ }^{\ddagger}$
}

\begin{abstract}
In this paper, we propose a two species competition model in a chemostat that uses a distributed delay to model the lag in the process of nutrient conversion and study the global asymptotic behavior of the model. The model includes a washout factor over the time delay involved in the nutrient conversion, and hence the delay is distributed over the species concentrations as well as over the nutrient concentration (using the gamma distribution). The results are valid for a very general class of monotone growth response functions.

By using the linear chain trick technique and the fluctuation lemma, we completely determine the global limiting behavior of the model, prove that there is always at most one survivor, and give a criterion to predict the outcome that is dependent upon the parameters in the delay kernel. We compare these predictions on the qualitative outcome of competition introduced by including distributed delay in the model with the predictions made by the corresponding discrete delay model, as well as with the corresponding no delay ODEs model. We show that the discrete delay model and the corresponding ODEs model can be obtained as limiting cases of the distributed delay models. Also, provided that the mean delays are small, the predictions of the delay models are almost identical with the predictions given by the ODEs model. However, when the mean delays are significant, the predictions given by the delay models concerning which species wins the competition and avoids extinction can be different from each other or from the predictions of the corresponding ODEs model. By varying the parameters in the delay kernels, we find that the model seems to have more potential to mimic reality. For example, computer simulations indicate that the larger the mean delay of the losing species, the more quickly that species proceeds toward extinction.
\end{abstract}

Key words. distributed delay, competition, chemostat, competitive exclusion, global asymptotic behavior

AMS subject classifications. 34D20, 34K20, 45M10, 92D25

\section{PII. S0036139995289842}

1. Introduction. Mathematical modeling has played a central role in many theoretical and experimental investigations of the chemostat, a device used for the continuous culture of microorganisms. A derivation of the basic chemostat equations,

$$
\begin{aligned}
S^{\prime}(t) & =\frac{F}{V}\left(S^{0}-S(t)\right)-N_{1}(t) \frac{p_{1}(S(t))}{c_{1}}-N_{2}(t) \frac{p_{2}(S(t))}{c_{2}}, \\
N_{1}^{\prime}(t) & =N_{1}(t)\left(-\frac{F}{V}+p_{1}(S(t))\right) \\
N_{2}^{\prime}(t) & =N_{2}(t)\left(-\frac{F}{V}+p_{2}(S(t))\right)
\end{aligned}
$$

describing exploitative competition by two populations of microorganisms for a single, essential, nonreproducing, growth-limiting nutrient, input at a constant rate can be found in Herbert, Elsworth, and Telling [21].

* Received by the editors August 2, 1995; accepted for publication (in revised form) June 24, 1996. http://www.siam.org/journals/siap/57-5/28984.html

$\dagger$ Department of Mathematics and Statistics, McMaster University, Hamilton, ON, Canada L8S 4K1 (wolkowic@mcmaster.ca, xia@daniel.math.mcmaster.ca). The research of the first author was supported by the Natural Sciences and Engineering Research Council of Canada.

$¥$ Department of Mathematics, Statistics and Computing Science, Dalhousie University, Halifax, NS, Canada B3H 3J5 (ruan@cs.dal.ca). The research of this author was supported by the Natural Sciences and Engineering Research Council of Canada. 
In model (1.1), $S(t)$ denotes the concentration of nutrient and $N_{i}(t)$ denotes the density of the $i$ th population of microorganisms in the culture vessel at time $t$. The parameter $V$ denotes the volume of the culture vessel and $F$ denotes the intake/output flow rate, so $\frac{F}{V}$ is the dilution rate. The concentration of the input nutrient in the feed vessel is denoted by $S^{0}$. The specific growth rate for each population is assumed to be a function of the nutrient concentration and is denoted by $p_{i}(S(t))$. The consumption rate is given by $\frac{p_{i}(S(t))}{c_{i}}$ and hence is assumed to be proportional to the specific growth rate, with constant of proportionality given by $c_{i}$, called the growth yield constant. The culture vessel is assumed to be well stirred. Species-specific death rates are assumed to be insignificant compared to the dilution rate and are ignored.

The global analysis of these equations was given by Hsu, Hubbell, and Waltman [25] and Hsu [24], in the case of response functions of the Michaelis-Menten form, often called the Monod model (see [37]). See Butler and Wolkowicz [7] and Wolkowicz and $\mathrm{Lu}$ [47] for a global analysis involving a more general class of response functions. See also Smith and Waltman [40] for a review of mathematical results on the theory of the chemostat. The global stability properties of the steady states derived from model (1.1) have led to interesting ecological predictions (see [21]). In particular, the model predicts that at most one population avoids extinction and that it is the relative values of the break-even concentrations that completely determine the outcome, dispelling the widely believed notion from classical competition theory (see Strobeck [42]) that the outcome of competition is independent of the intrinsic rates of increase of the two species. Also, the model predicts that this qualitative outcome is independent of the growth yield constants. Motivated by the mathematical predictions in [25], Hansen and Hubbell [20] carried out experiments that seemed to demonstrate the usefulness of these break-even concentrations in predicting the qualitative outcome. On the other hand, they noticed that the losing population had a faster death rate in their experiments than the model predicted and that there seemed to be more oscillations in the transients. Tilman [45] provides interesting theoretical, experimental, and correlational information on the chemostat.

Others have also noticed experimental deviations of a quantitative nature from theoretical predictions based on the Monod model. Jannasch and Mateles [27] and Veldkamp [46] mentioned that the yield constants in practice do not seem to be constant and pointed out that this might account for the discrepancy. This was extensively discussed in the work of Droop [14], who observed that, under nonequilibrium conditions, the nutrient concentration in the chemostat remained relatively high at low dilution rates, an anomaly that cannot be explained by the Monod equations. This led him to introduce the notion of an internal nutrient pool and the formulation of the variable-yield model. According to Droop, only the internal nutrient is immediately available for cell growth, and passage of nutrient from outside to inside the cells introduces inevitable time delays. Thus the assumption that the external nutrient supply is instantaneously converted to biomass is a broad oversimplification and should (at least in part) account for the inadequacies of the Monod model in nonequilibrium situations. See Smith and Waltman [40] for a global analysis of this model.

The recognition of time delay in the growth response of a population to changes in the environment has led to extensive experimental and theoretical studies. To better fit his observed data, Caperon [8] introduced a discrete delay as well as a distributed delay in one of the equations of the Monod chemostat model. The model used 
by Caperon [8], however, allowed the nutrient concentration to take negative values. This problem with the model was corrected by Thingstad and Langeland [44], who adopted the original Monod model and incorporated a discrete delay in the species growth equation. Bush and Cook [6] considered the same discrete delay model, but with inhibitory growth response functions. In an attempt to analyze the existing laboratory data, MacDonald [31] considered distributed delay models and discussed them in terms of linear stability. Delays were also introduced in Droop's model by Cunningham and Nisbet [11] in order to understand the observed transient behavior following sharp changes in dilution rate. We refer to MacDonald [33] for a thorough survey of earlier work on chemostat models with time delay. See also Freedman, So, and Waltman [16] for a model that predicts that oscillatory coexistence of two competing species in a chemostat is possible. We remark that most of the delay models mentioned in this paragraph permit nontrivial periodic solutions when delays are increased beyond a threshold. This is not surprising, because time delay is often considered to be a source of oscillations. However, as MacDonald [33] points out, empirical evidence for sustained, undamped oscillations in simple chemostat experiments has been rather sporadic. One usually observes strongly damped oscillations under nonequilibrium conditions (see, for example, [8]). See Cunningham and Nisbet [11] for a discussion on the difficulties involved in introducing delays in chemostat models.

Recent attempts have been made to incorporate delays more appropriately in chemostat models. In [17], Freedman, So, and Waltman consider a different discrete delay model than the one they considered in [16]. What distinguishes this model from the earlier delay chemostat models is the inclusion of a washout factor over the time of the delay, and hence the delays appear simultaneously in the variables of nutrient and species concentrations. Using the same notation as in model (1.1), with $D=\frac{F}{V}$ the model takes the form

$$
\begin{aligned}
& S^{\prime}(t)=D\left(S^{0}-S(t)\right)-x_{1}(t) p_{1}(S(t))-x_{2}(t) p_{2}(S(t)), \\
& x_{1}^{\prime}(t)=-D x_{1}(t)+e^{-D \tau_{1}} x_{1}\left(t-\tau_{1}\right) p_{1}\left(S\left(t-\tau_{1}\right)\right), \\
& x_{2}^{\prime}(t)=-D x_{2}(t)+e^{-D \tau_{2}} x_{2}\left(t-\tau_{2}\right) p_{2}\left(S\left(t-\tau_{2}\right)\right),
\end{aligned}
$$

where for convenience the yield constants have been scaled out by letting $x_{i}(t)=\frac{N_{i}(t)}{c_{i}}$. Each constant $\tau_{i} \geq 0$ represents the time delay involved in the conversion of nutrient to viable cells. Due to the outflow in the chemostat, only $e^{-D \tau_{i}} x_{i}\left(t-\tau_{i}\right)$, not $x_{i}(t)$, of the $x_{i}\left(t-\tau_{i}\right)$ microorganisms that consumed nutrient $\tau_{i}$ units of time previously survive in the chemostat the $\tau_{i}$ units of time assumed necessary to complete the process of converting the nutrient to new cells.

Model (1.2) appears to be more reasonable than the previous model considered in [16]. It was also studied by Ellermeyer [15], Hsu, Waltman, and Ellermeyer [26], and Wolkowicz and Xia [48]. In [48], it was shown that under the assumptions that the response functions $p_{i}$ are monotone increasing and locally Lipschitz, model (1.2) generates parameters $\lambda_{i}\left(\tau_{i}\right)$ that depend on the delays, are analogues of the break-even concentrations $\left(\lambda_{i}=\lambda_{i}(0)\right)$ in model (1.1), and, as for (1.1), completely determine the competitive ability of each species. Also, as for (1.1), under the generic condition that $\lambda_{1}\left(\tau_{1}\right) \neq \lambda_{2}\left(\tau_{2}\right)$, at most one population avoids extinction and all concentrations eventually equilibrate. Thus the global asymptotic behavior of (1.2) is fully understood. However, as Wolkowicz and Xia [48] point out, since the criteria that determine competitive ability depend on the delays, if at least one of the delays $\tau_{i}$ is large enough, 
for fixed $p_{i}$ and $p_{j}$, it is possible that $\lambda_{i}(0)<\lambda_{j}(0)$ but that $\lambda_{j}\left(\tau_{j}\right)<\lambda_{i}\left(\tau_{i}\right)$; that is, the relative values of the $\lambda_{k}\left(\tau_{k}\right)$ can be reversed, and so model (1.2) can predict the opposite outcome of competition predicted by the corresponding ODEs model. See [48] for a detailed mathematical analysis of model (1.2) that also covers the $n$-species competition model and allows for differential death rates.

The idea of taking into account the washout factor over the time delay in chemostat models is not new and was seen, for example, in Levin, Stewart, and Chao [30], who studied a discrete delay competition model of bacteria (prey) and bacteriophage (predator) in the chemostat. Since delay occurs between the attack of bacteriophages and the appearance of new bacteriophages, only those bacteria and bacteriophages not washed out during the delay need to be considered. Even before that, Dietz, Molineaux, and Thomas [13] included an approximation of the exponential factor (in fact, $(1-D)^{\tau}$, where $D$ is the death rate of the populations concerned and $\tau$ is the incubation period) in an epidemiological model of malaria. Because the lifetime of infective individuals may be shorter than the incubation period, the factor is needed to represent the approximate proportion of newly infected individuals who survive during the incubation period (see also Aron and May [1] and Bailey [2]). MacDonald $[33,34]$ also discusses this idea.

As is well known, in many biological models, the assumption that the time delay is discrete as in model (1.2) is often an oversimplification. It is the purpose of this paper to propose and analyze a more realistic model in which a distributed delay is incorporated. The model is based on (1.1) and, as suggested by MacDonald [33], includes the washout rate in the delay kernel as in (1.2). We study the global asymptotic behavior of the solutions and draw conclusions about the effect distributed delay has on the qualitative outcome of competition. We choose a special family of generic delay kernels, the gamma distribution, to model the delay. As for models (1.1) and (1.2), there are analogous parameters $\lambda_{1}$ and $\lambda_{2}$ depending on parameters in the delay kernel that completely determine the competitive ability of each species. As in models (1.1) and (1.2), provided the generic condition $\lambda_{1} \neq \lambda_{2}$ holds, there is always at most one competitor population that avoids extinction, and all solutions eventually equilibrate. And as for model (1.2), reversal of the prediction of outcome compared to model (1.1) is possible for appropriate members of the family of delay kernels. Also, our computer simulations seem to indicate that the delay can influence how quickly a population approaches extinction or how quickly its concentration equilibrates. Solutions can also have slightly more transient oscillations than solutions of the instantaneous model. We thus believe that our model may more accurately mimic the dynamic behavior of pure and simple competition in the chemostat and hence help to explain some of the experimental discrepancies from previous theory. Also, it is interesting to note that it is possible to obtain models (1.1) and (1.2) as limiting cases of the model proposed here, and in this sense our model represents a unifying framework.

Distributed delay (or integrodifferential equation) models have been used in biological modeling since the work of Volterra (see Scudo and Ziegler [39]), and they are considered to be more realistic than discrete delay models (see, for example, Caperon [8], Caswell [9], and May [35]). The monographs of Cushing [12] and MacDonald [32] give excellent descriptions of models involving integrodifferential equations and discuss their mathematical analysis. However, there has been relatively little emphasis on including distributed delay in chemostat models (see Caperon [8], MacDonald [31], Ruan and Wolkowicz [38], and Stephens and Lyberatos [41]). Although global results 
are known for many other population models involving distributed delay (see Gopalsamy [18] and Kuang [28]), there are few results on the global asymptotic behavior of such models in the chemostat (see Beretta and Takeuchi [3] for some interesting results on chemostat-like models with nutrient recycling). To the best of our knowledge, the results in this paper appear to be the first global results on the basic chemostat model involving distributed delay. Our proofs are based on the linear chain trick technique (see MacDonald [32]) and the fluctuation lemma (see Hirsch, Hanisch, and Gabriel [23]). We avoid the local stability analysis and the theory of asymptotically autonomous differential equations (see Thieme [43]) that are often used in analyzing chemostat models.

This paper is organized as follows. The model is described in section 2 . In section 3, we state the main results. The proofs are carried out in section 4 . Some observations based on computer simulations are given in section 5 . We conclude the paper with a discussion in section 6 . Finally, in the appendix we prove that models (1.1) and (1.2) are limiting cases of the proposed distributed delay model.

2. The model and preliminary analysis. We consider the following modification of the basic chemostat model (1.1) that includes a distributed delay to describe the delay involved in the process of conversion of nutrient consumed into viable cells. As for model (1.2), we use the same notation as in model (1.1), with $D=\frac{F}{V}$, and the yield constants scaled out by letting $x_{i}(t)=\frac{N_{i}(t)}{c_{i}}$. The model takes the form

$$
\begin{aligned}
& S^{\prime}(t)=\left(S^{0}-S(t)\right) D-x_{1}(t) p_{1}(S(t))-x_{2}(t) p_{2}(S(t)), \\
& x_{1}^{\prime}(t)=-D x_{1}(t)+\int_{-\infty}^{t} x_{1}(\theta) p_{1}(S(\theta)) e^{-D(t-\theta)} K_{1}(t-\theta) d \theta, \\
& x_{2}^{\prime}(t)=-D x_{2}(t)+\int_{-\infty}^{t} x_{2}(\theta) p_{2}(S(\theta)) e^{-D(t-\theta)} K_{2}(t-\theta) d \theta .
\end{aligned}
$$

$K_{i}: \mathbb{R}_{+} \rightarrow \mathbb{R}_{+}$denote the delay kernels. Throughout this paper, we use the family of generic delay kernels of the form

$$
K_{i}(u)=\frac{\alpha_{i}^{r_{i}+1} u^{r_{i}}}{r_{i} !} e^{-\alpha_{i} u}, \quad i=1,2,
$$

where the $\alpha_{i}>0$ are constants and the $r_{i} \geq 0$ are integers. According to MacDonald [32], $r_{i}$ is called the order of the delay kernel $K_{i}$ and the mean delay corresponding to the $K_{i}$ is

$$
\tau_{i}=\int_{0}^{\infty} u K_{i}(u) d u=\frac{r_{i}+1}{\alpha_{i}} .
$$

In the literature, the kernels $K_{i}(u)$ with $r_{i}=0$ and $r_{i}=1$ are called the weak and strong kernels, respectively, and are frequently used in biological modeling (see Cushing [12], MacDonald [32]). It is worth noting that the discrete delay model (1.2) can be viewed as a limiting case of model (2.1) by letting the $r_{i}$ go to infinity while keeping the mean delays $\frac{r_{i}+1}{\alpha_{i}}$ fixed (see Appendix).

We shall study the global asymptotic behavior of model (2.1) under the following assumptions concerning the nutrient uptake functions $p_{i}, i=1,2$ :

$$
p_{i}: \mathbb{R}_{+} \rightarrow \mathbb{R}_{+} \text {is monotone increasing and locally Lipschitz with } p_{i}(0)=0 ;
$$


there exists a positive (possibly extended) real number $\lambda_{i}$ such that

$$
\begin{array}{ll}
p_{i}(S)<D\left(\frac{D+\alpha_{i}}{\alpha_{i}}\right)^{r_{i}+1} & \text { if } S<\lambda_{i}, \\
p_{i}(S)>D\left(\frac{D+\alpha_{i}}{\alpha_{i}}\right)^{r_{i}+1} & \text { if } S>\lambda_{i} .
\end{array}
$$

Under assumptions (2.3) and (2.4), system (2.1) always has the washout equilibrium $E_{0}=\left(S^{0}, 0,0\right)$. Moreover, for each $i$ such that $\lambda_{i}<S^{0}$, there is an equilibrium of the form

$$
\begin{aligned}
& E_{1}=\left(\lambda_{1},\left(\frac{\alpha_{1}}{D+\alpha_{1}}\right)^{r_{1}+1}\left(S^{0}-\lambda_{1}\right), 0\right) \quad \text { if } i=1, \\
& E_{2}=\left(\lambda_{2}, 0,\left(\frac{\alpha_{2}}{D+\alpha_{2}}\right)^{r_{2}+1}\left(S^{0}-\lambda_{2}\right)\right) \quad \text { if } i=2 .
\end{aligned}
$$

Note that the presence of the washout memory factor in model (2.1) changes the equilibrium values for the corresponding ODEs model. Therefore, the equilibria $E_{i}, i=1,2$, differ from those when delays and washout effects are ignored.

Let $B C_{+}^{3}$ denote the Banach space of bounded continuous functions mapping from $(-\infty, 0]$ to $\mathbb{R}_{+}^{3}$. From the general theory of integral differential equations (see Burton [4] and Miller [36]), for any initial data $\phi=\left(\phi_{0}, \phi_{1}, \phi_{2}\right) \in B C_{+}^{3}$, there exists a unique solution $\pi(\phi ; t):=\left(S(\phi ; t), x_{1}(\phi ; t), x_{2}(\phi ; t)\right)$ for all $t \geq 0$ and $\left.\pi(\phi ; \cdot)\right|_{(-\infty, 0]}=\phi$. Throughout, we will also use $\left(S(t), x_{1}(t), x_{2}(t)\right)$ to denote the solution $\pi(\phi ; t)$ with $\phi \in$ $B C_{+}^{3}$, when no confusion arises. By a positive solution $\pi(\phi ; t)$ or $\left(S(t), x_{1}(t), x_{2}(t)\right)$ of (2.1), we shall mean the solution has initial data $\phi \in B C_{+}^{3}$ and each component of the solution is positive for all $t>0$.

In this section, we give two preliminary results about model (2.1). The first one indicates that model (2.1) possesses the property that positive initial data yield positive solutions.

LEMMA 2.1 (positivity lemma). For any $\phi \in B C_{+}^{3}$ with $\phi_{0}(0) \geq 0$ and $\phi_{i}(0)>$ $0, i=1,2$, the solution $\pi(\phi ; t)$ remains positive for all $t>0$.

Proof. If $S(\xi)=0$ for some $\xi \geq 0$, the first equation of $(2.1)$ gives $S^{\prime}(\xi)>0$. This implies $S(\phi ; t)>0$ for all $t>0$. To show that $x_{i}(\phi ; t)>0$ for all $t>0$, we suppose, to the contrary, that it is not true. Let $\bar{t}=\inf \left\{t>0 ; x_{i}(\phi ; t)=0\right.$ and $x_{i}(\theta)>0$ for $0 \leq \theta<t\}<\infty$. Then $x_{i}(\bar{t})=0$ and $x_{i}^{\prime}(\bar{t}) \leq 0$. But, from (2.1), we have

$$
\begin{aligned}
x_{i}^{\prime}(\bar{t}) & =-D x_{i}(\bar{t})+\int_{-\infty}^{\bar{t}} x_{i}(\theta) p_{i}(S(\theta)) e^{-D(\bar{t}-\theta)} K_{i}(\bar{t}-\theta) d \theta \\
& =\int_{-\infty}^{\bar{t}} x_{i}(\theta) p_{i}(S(\theta)) e^{-D(\bar{t}-\theta)} K_{i}(\bar{t}-\theta) d \theta>0 .
\end{aligned}
$$

This is a contradiction. Therefore, $x_{i}(\phi ; t)>0$ for any $t>0$.

Analogous to models (1.1) and (1.2), system (2.1) is also dissipative in the sense that every positive solution is bounded.

LEMMA 2.2 (dissipativity lemma). All positive solutions of model (2.1) are bounded for $t>0$.

Proof. Recalling that the delay kernels are of the form (2.2), we use the linear chain trick technique (see MacDonald [32]). Let $\left(S(t), x_{1}(t), x_{2}(t)\right)$ be any fixed 
positive solution of (2.1). We define

$$
\begin{aligned}
& y_{i}(t)=\int_{-\infty}^{t} x_{1}(\theta) p_{1}(S(\theta)) G_{D, \alpha_{1}}^{i}(t-\theta) d \theta, \quad i=0,1, \ldots, r_{1}, \\
& z_{j}(t)=\int_{-\infty}^{t} x_{2}(\theta) p_{2}(S(\theta)) G_{D, \alpha_{2}}^{j}(t-\theta) d \theta, \quad j=0,1, \ldots, r_{2},
\end{aligned}
$$

where

$$
G_{D, \alpha_{i}}^{k}(t)=\frac{\alpha_{i}^{k+1}}{k !} t^{k} e^{-\left(D+\alpha_{i}\right) t}, \quad i=1,2 \quad \text { and } k=0,1, \ldots, \max \left(r_{1}, r_{2}\right) .
$$

Observe that for any $k \geq 1, i=1,2$,

$$
\begin{aligned}
\frac{d}{d t} G_{D, \alpha_{i}}^{k}(t) & =\alpha_{i} G_{D, \alpha_{i}}^{k-1}(t)-\left(D+\alpha_{i}\right) G_{D, \alpha_{i}}^{k}(t), \\
\frac{d}{d t} G_{D, \alpha_{i}}^{0}(t) & =-\left(D+\alpha_{i}\right) G_{D, \alpha_{i}}^{0}(t) .
\end{aligned}
$$

We find that $\left(S(t), x_{1}(t), y_{0}(t), \ldots, y_{r_{1}}(t), x_{2}(t), z_{0}(t), \ldots, z_{r_{2}}(t)\right)$ satisfies

$$
\begin{aligned}
S^{\prime} & =\left(S^{0}-S\right) D-x_{1} p_{1}(S)-x_{2} p_{2}(S), \\
x_{1}^{\prime} & =-D x_{1}+y_{r_{1}}, \\
y_{0}^{\prime} & =-\left(D+\alpha_{1}\right) y_{0}+\alpha_{1} x_{1} p_{1}(S), \\
y_{i}^{\prime} & =-\left(D+\alpha_{1}\right) y_{i}+\alpha_{1} y_{i-1}, \quad i=1,2, \ldots, r_{1}, \\
x_{2}^{\prime} & =-D x_{2}+z_{r_{2}}, \\
z_{0}^{\prime} & =-\left(D+\alpha_{2}\right) z_{0}+\alpha_{2} x_{2} p_{2}(S), \\
z_{j}^{\prime} & =-\left(D+\alpha_{2}\right) z_{j}+\alpha_{2} z_{j-1}, \quad j=1,2, \ldots, r_{2} .
\end{aligned}
$$

Let

$$
W(t)=S^{0}-S(t)-\sum_{i=0}^{r_{1}} \frac{y_{i}(t)}{\alpha_{1}}-\sum_{j=0}^{r_{2}} \frac{z_{j}(t)}{\alpha_{2}}-x_{1}(t)-x_{2}(t), \quad t \geq 0 .
$$

It follows from (2.6) that $W^{\prime}(t)=-D W(t), t \geq 0$, and therefore

$$
S(t)+\sum_{i=0}^{r_{1}} \frac{y_{i}(t)}{\alpha_{1}}+\sum_{j=0}^{r_{2}} \frac{z_{j}(t)}{\alpha_{2}}+x_{1}(t)+x_{2}(t)=S^{0}+\rho(t), \quad t \geq 0,
$$

where $\rho(t)$ is a continuous function and $\rho(t) \rightarrow 0$ exponentially as $t \rightarrow \infty$. Note that $y_{i}(t)$ and $z_{j}(t)$ are all positive for $t>0$. The boundedness of the solution $\left(S(t), x_{1}(t), x_{2}(t)\right)$ now follows immediately from (2.8).

3. Statement of results. In each of the theorems below, we assume that model (2.1) satisfies (2.2)-(2.4). Our results shall give a complete description of the global asymptotic behavior of model (2.1) under the generic condition $\lambda_{1} \neq \lambda_{2}$.

The first result states that if $\lambda_{i}$ for population $x_{i}$ is larger than the input nutrient concentration, that population approaches extinction whether or not there is a competitor. Thus the elimination of $x_{i}$ in this case has nothing to do with competition. 
THEOREM 3.1 (inadequate competitor). Let $\pi(\phi ; t)$ be any positive solution of (2.1). If $\lambda_{i} \geq S^{0}$ for some $i \in\{1,2\}$, then $x_{i}(\phi ; t) \rightarrow 0$ as $t \rightarrow \infty$.

Theorem 3.1 immediately implies the following global result that describes outcomes in which both populations are eliminated from the chemostat because the chemostat is an inadequate environment for either population to survive, rather than as a result of competition.

THEOREM 3.2. If $\lambda_{1}, \lambda_{2} \geq S^{0}$, then $\lim _{t \rightarrow \infty} \pi(\phi ; t)=E_{0}$ for every positive solution $\pi(\phi ; t)$ of $(2.1)$.

When only one of the $\lambda_{i}$ 's is smaller than $S^{0}$, we can also determine the global asymptotic behavior of positive solutions.

THEOREM 3.3. If $\lambda_{i}<S^{0} \leq \lambda_{j}, i \neq j$, then $\lim _{t \rightarrow \infty} \pi(\phi ; t)=E_{i}$ for every positive solution $\pi(\phi ; t)$ of $(2.1)$.

As we will see in the proofs of Theorems 3.1-3.3, when only one population $x_{1}$ is cultured in the chemostat, the necessary and sufficient condition for $x_{1}$ to survive is $\lambda_{1}<S^{0}$. The most interesting question is then whether both populations can coexist in the chemostat in the case that each population can survive in the absence of the other. In this case, we show that model (2.1) exhibits competitive exclusion and hence competition is the agent of elimination.

THEOREM 3.4 (competitive exclusion). If $\lambda_{i}<\lambda_{j}<S^{0}, i \neq j$, then $\lim _{t \rightarrow \infty} \pi(\phi ; t)=E_{i}$ for every positive solution of $(2.1)$.

4. Proofs of the results. We begin with the following elementary lemma due to Barbălat, which will be needed throughout this section. For a proof, see Gopalsamy [18].

LEMMA 4.1 (Barbălat lemma). Let a be a finite number and $f:[a, \infty) \rightarrow \mathbb{R}$ be $a$ differentiable function. If $\lim _{t \rightarrow \infty} f(t)$ exists (finite) and the derivative function $f^{\prime}$ is uniformly continuous on $(a, \infty)$, then $\lim _{t \rightarrow \infty} f^{\prime}(t)=0$.

Remark. This lemma will be useful, since if $\left(S(t), x_{1}(t), y_{0}(t), \ldots, y_{r_{1}}(t), x_{2}(t)\right.$, $\left.z_{0}(t), \ldots, z_{r_{2}}(t)\right)$ satisfies (2.6) for positive initial conditions, then each component of this solution vector and its derivative function is a uniformly continuous function on $[0, \infty)$. To see this, note that by $(2.8)$, it follows that all of the components of this solution vector are bounded functions on $[0, \infty)$. Therefore, all of their derivatives are continuous and bounded functions on $[0, \infty)$, as they are defined by (2.6). Applying the mean value theorem, all the component functions are thus Lipschitz continuous and hence uniformly continuous. Since $S(t)$ is bounded, let $M=\sup _{t \geq 0} S(t)<\infty$. Then, each function $p_{i}, i=1,2$, is uniformly continuous on $[0, M]$. By $(2.6)$, the derivative of each component of the solution vector is defined as the sum, difference, product and composition of uniformly continuous functions and hence, is also uniformly continuous.

Proof of Theorem 3.1. Without loss of generality, we assume that $\lambda_{1} \geq S^{0}$. Let $y_{i}(t)$ be defined as in (2.5). It follows from $(2.6)$ that $\left(S(t), x_{1}(t), y_{0}(t), \ldots, y_{r_{1}}(t)\right)$ satisfies

$$
\begin{aligned}
& S^{\prime}=\left(S^{0}-S\right) D-x_{1} p_{1}(S)-x_{2} p_{2}(S), \\
& x_{1}^{\prime}=-D x_{1}+y_{r_{1}}, \\
& y_{0}^{\prime}=-\left(D+\alpha_{1}\right) y_{0}+\alpha_{1} x_{1} p_{1}(S), \\
& y_{i}^{\prime}=-\left(D+\alpha_{1}\right) y_{i}+\alpha_{1} y_{i-1}, \quad i=1,2, \ldots, r_{1} .
\end{aligned}
$$


Define

$$
w(t)=\sum_{i=0}^{r_{1}} \frac{\left(D+\alpha_{1}\right)^{i}}{\alpha_{1}^{i+1}} y_{i}(t)+\left(\frac{D+\alpha_{1}}{\alpha_{1}}\right)^{r_{1}+1} x_{1}(t), \quad t \geq 0 .
$$

It follows from (2.5) and (2.8) that $w(t)$ is positive and bounded for $t>0$. Moreover, by $(4.1)$,

$$
w^{\prime}(t)=x_{1}(t)\left[-D\left(\frac{D+\alpha_{1}}{\alpha_{1}}\right)^{r_{1}+1}+p_{1}(S(t))\right] .
$$

On the other hand, from the first equation of (4.1), we conclude that either $S(t) \downarrow S^{0}$ as $t \rightarrow \infty$ or $S(t)<S^{0}$ for all large $t$.

Suppose that we have $S(t) \downarrow S^{0}$ as $t \rightarrow \infty$. Then, $\lim _{t \rightarrow \infty} x_{1}(t)=0$ follows immediately from (2.8), as desired.

Now suppose that $S(t)<S^{0} \leq \lambda_{1}$ for all large $t$. In this case, (4.3) gives $w^{\prime}(t)<0$ for large $t$. Thus $w(t) \downarrow w^{*}$ as $t \rightarrow \infty$ for some $w^{*} \geq 0$. Note that (2.8) holds. It follows from (4.1) and the remark following Lemma 4.1 that the functions $y_{i}^{\prime}(t)$ and $x_{1}^{\prime}(t)$ are all uniformly continuous. Hence $w^{\prime}(t)$ is also uniformly continuous. By Lemma 4.1, $\lim _{t \rightarrow \infty} w^{\prime}(t)=0$. Now we use (4.3) to obtain

$$
\lim _{t \rightarrow \infty} x_{1}(t)\left[-D\left(\frac{D+\alpha_{1}}{\alpha_{1}}\right)^{r_{1}+1}+p_{1}(S(t))\right]=0 .
$$

If $\lim \sup _{t \rightarrow \infty} x_{1}(t)>0$, then $\lim _{m \rightarrow \infty} x_{1}\left(t_{m}\right)=\gamma$ for some sequence $\left\{t_{m}\right\} \uparrow \infty$ and real number $\gamma>0$. By (4.4), we have $\lim _{m \rightarrow \infty} S\left(t_{m}\right)=\lambda_{1}$. This is a contradiction if $\lambda_{1}>S^{0}$. When $\lambda_{1}=S^{0}$, we use (2.8) to obtain $\lim _{m \rightarrow \infty} x_{1}\left(t_{m}\right)=0$, contradicting $\gamma>0$. Therefore $\limsup _{t \rightarrow \infty} x_{1}(t)=0$ and the conclusion follows.

Proof of Theorem 3.2. We first observe that by Theorem $3.1, \lim _{t \rightarrow \infty} x_{i}(t)=0$ for $i=1,2$. Define $y_{i}(t)$ and $z_{j}(t)$ as in $(2.5)$. Then $\left(S(t), x_{1}(t), y_{0}(t), \ldots, y_{r_{1}}(t), x_{2}(t)\right.$, $\left.z_{0}(t), \ldots, z_{r_{2}}(t)\right)$ satisfies (2.6). By using the boundedness of the solution, Lemma 4.1, and the equations in (2.6), it is not difficult to see that $\lim _{t \rightarrow \infty} y_{i}(t)=0$ for all $i=0,1,2, \ldots, r_{1}$ and $\lim _{t \rightarrow \infty} z_{j}(t)=0$ for all $j=0,1,2, \ldots, r_{2}$. According to (2.8), we then obtain $\lim _{t \rightarrow \infty} S(t)=S^{0}$. Therefore $\lim _{t \rightarrow \infty}\left(S(t), x_{1}(t), x_{2}(t)\right)=E_{0}$.

To prove Theorems 3.3 and 3.4, we first study system (2.6). By using (2.8), it suffices to consider the following reduced system of asymptotically autonomous differential equations:

$$
\begin{aligned}
& x_{1}^{\prime}=-D x_{1}+y_{r_{1}}, \\
& y_{0}^{\prime}=-\left(D+\alpha_{1}\right) y_{0}+\alpha_{1} x_{1} p_{1}\left(S^{0}-\sum_{i=0}^{r_{1}} \frac{y_{i}}{\alpha_{1}}-\sum_{j=0}^{r_{2}} \frac{z_{j}}{\alpha_{2}}-x_{1}-x_{2}+\rho(t)\right), \\
& y_{i}^{\prime}=-\left(D+\alpha_{1}\right) y_{i}+\alpha_{1} y_{i-1}, \quad i=1,2, \ldots, r_{1}, \\
& x_{2}^{\prime}=-D x_{2}+z_{r_{2}}, \\
& z_{0}^{\prime}=-\left(D+\alpha_{2}\right) z_{0}+\alpha_{2} x_{2} p_{2}\left(S^{0}-\sum_{i=0}^{r_{1}} \frac{y_{i}}{\alpha_{1}}-\sum_{j=0}^{r_{2}} \frac{z_{j}}{\alpha_{2}}-x_{1}-x_{2}+\rho(t)\right), \\
& z_{j}^{\prime}=-\left(D+\alpha_{2}\right) z_{j}+\alpha_{2} z_{j-1}, \quad j=1,2, \ldots, r_{2},
\end{aligned}
$$


where $\rho(t)$ is continuous and $\rho(t) \rightarrow 0$ exponentially as $t \rightarrow \infty$. For the sake of convenience, we rewrite (4.5) in another form by introducing new variables

$$
\begin{array}{ll}
u_{i}(t)=x_{1}(t)+\sum_{j=i}^{r_{1}} \frac{y_{j}(t)}{\alpha_{1}}, & i=0,1, \ldots, r_{1}+1, \\
v_{i}(t)=x_{2}(t)+\sum_{j=i}^{r_{2}} \frac{z_{j}(t)}{\alpha_{2}}, & i=0,1, \ldots, r_{2}+1,
\end{array}
$$

where we use the convention that $\sum_{j=m}^{n} k_{j} \equiv 0$ if $n<m$. Thus, $x_{1} \equiv u_{r_{1}+1}$ and $x_{2} \equiv v_{r_{2}+1}$. By using (4.6), we obtain a new system as follows

$$
\begin{aligned}
& u_{0}^{\prime}(t)=-D u_{0}(t)+u_{r_{1}+1}(t) p_{1}\left(S^{0}-u_{0}(t)-v_{0}(t)+\rho(t)\right), \\
& u_{i}^{\prime}(t)=-\left(D+\alpha_{1}\right) u_{i}(t)+\alpha_{1} u_{i-1}(t), \quad i=1,2, \ldots, r_{1}+1, \\
& v_{0}^{\prime}(t)=-D v_{0}(t)+v_{r_{2}+1}(t) p_{2}\left(S^{0}-u_{0}(t)-v_{0}(t)+\rho(t)\right), \\
& v_{j}^{\prime}(t)=-\left(D+\alpha_{2}\right) v_{j}(t)+\alpha_{2} v_{j-1}(t), \quad j=1,2, \ldots, r_{2}+1 .
\end{aligned}
$$

If $\left(S(t), x_{1}(t), x_{2}(t)\right)$ is a positive solution of (2.1), then by Lemma 2.2, together with (2.5), (2.8), and (4.6), $u_{i}(t)$ and $v_{j}(t)$ are all positive and bounded. Therefore, the following numbers are well defined:

$$
\begin{gathered}
\delta_{i}=\liminf _{t \rightarrow \infty} u_{i}(t), \quad \gamma_{i}=\limsup _{t \rightarrow \infty} u_{i}(t), \quad i=0,1, \ldots, r_{1}+1, \\
a_{j}=\liminf _{t \rightarrow \infty} v_{j}(t), \quad b_{j}=\limsup _{t \rightarrow \infty} v_{j}(t), \quad j=0,1, \ldots, r_{2}+1 .
\end{gathered}
$$

Clearly, $0 \leq \delta_{i} \leq \gamma_{i}$ and $0 \leq a_{j} \leq b_{j}$ for all $i \in\left\{0,1, \ldots, r_{1}+1\right\}$ and $j \in\left\{0,1, \ldots, r_{2}+\right.$ 1\}. Moreover, it follows from the remark following Lemma 4.1, that $u_{i}(t), v_{j}(t)$ and their derivatives $u_{i}^{\prime}(t), v_{j}^{\prime}(t)$ are also uniformly continuous on $[0, \infty)$.

To proceed further, we need the following useful lemma due to Hirsch, Hanisch, and Gabriel [23].

LEMMA 4.2 (fluctuation lemma). If $f: \mathbb{R}^{+} \rightarrow \mathbb{R}$ is a differentiable function and $\liminf \operatorname{in}_{t \rightarrow \infty} f(t)<\limsup _{t \rightarrow \infty} f(t)$, then there exist sequences $\left\{t_{m}\right\} \uparrow \infty$ and $\left\{s_{m}\right\} \uparrow \infty$ such that

$$
\begin{aligned}
& \lim _{m \rightarrow \infty} f\left(t_{m}\right)=\limsup _{t \rightarrow \infty} f(t), \quad f^{\prime}\left(t_{m}\right)=0, \\
& \lim _{m \rightarrow \infty} f\left(s_{m}\right)=\liminf _{t \rightarrow \infty} f(t), \quad f^{\prime}\left(s_{m}\right)=0 .
\end{aligned}
$$

In what follows, we assume that $\left(S(t), x_{1}(t), x_{2}(t)\right)$ is an arbitrarily fixed positive solution of (2.1) and $y_{i}(t), z_{j}(t), u_{i}(t), v_{j}(t), \delta_{i}, \gamma_{i}, a_{j}, b_{j}$ are defined as in (2.5), (4.6) and (4.8). Recall that $y_{i}(t), z_{j}(t)$ and $u_{i}(t), v_{j}(t)$ are all positive and satisfy (4.5) and (4.7), respectively.

The proofs of Theorems 3.3 and 3.4 will follow from the following five lemmas.

LEMMA 4.3. We have

$$
\left(\frac{\alpha_{1}}{D+\alpha_{1}}\right)^{i} \delta_{0} \leq \delta_{i} \leq \gamma_{i} \leq\left(\frac{\alpha_{1}}{D+\alpha_{1}}\right)^{i} \gamma_{0}, \quad i=1,2, \ldots, r_{1}+1
$$

and

$$
\left(\frac{\alpha_{2}}{D+\alpha_{2}}\right)^{j} a_{0} \leq a_{j} \leq b_{j} \leq\left(\frac{\alpha_{2}}{D+\alpha_{2}}\right)^{j} b_{0}, \quad j=1,2, \ldots, r_{2}+1
$$


Proof. We first show that

$$
\gamma_{i} \leq \frac{\alpha_{1}}{D+\alpha_{1}} \gamma_{i-1}, \quad i=1,2, \ldots, r_{1}+1
$$

We consider two cases.

Case 1. $\delta_{i}<\gamma_{i}$. We apply Lemma 4.2 to obtain a sequence $\left\{t_{m}\right\} \uparrow \infty$ such that

$$
\lim _{m \rightarrow \infty} u_{i}\left(t_{m}\right)=\gamma_{i} \quad \text { and } \quad u_{i}^{\prime}\left(t_{m}\right)=0
$$

for all $m$. By (4.7), we have

$$
\left(D+\alpha_{1}\right) u_{i}\left(t_{m}\right)=\alpha_{i} u_{i-1}\left(t_{m}\right) .
$$

Taking the limit $(m \rightarrow \infty)$ on both sides leads to (4.11).

Case 2. $\delta_{i}=\gamma_{i}$. In this case, we apply Lemma 4.1 to obtain $\lim _{t \rightarrow \infty} u_{i}^{\prime}(t)=0$.

Using (4.7) once more, we have

$$
\lim _{t \rightarrow \infty}\left[-\left(D+\alpha_{1}\right) u_{i}(t)+\alpha_{1} u_{i-1}(t)\right]=0,
$$

which gives

$$
\gamma_{i}=\lim _{t \rightarrow \infty} u_{i}(t)=\lim _{t \rightarrow \infty} \frac{\alpha_{1}}{D+\alpha_{1}} u_{i-1}(t)=\frac{\alpha_{1}}{D+\alpha_{1}} \gamma_{i-1}
$$

as desired.

It now follows from (4.11) that

$$
\gamma_{i} \leq\left(\frac{\alpha_{1}}{D+\alpha_{1}}\right)^{i} \gamma_{0}, \quad i=1,2, \ldots, r_{1}+1
$$

Similarly, one can show that

$$
\delta_{i} \geq \frac{\alpha_{1}}{D+\alpha_{1}} \delta_{i-1} \geq\left(\frac{\alpha_{1}}{D+\alpha_{1}}\right)^{i} \delta_{0}, \quad i=1,2, \ldots, r_{1}+1 .
$$

Combining (4.12) and (4.13) then gives (4.9). The proof of (4.10) is similar and we omit the details.

LEMMA 4.4. $\gamma_{0} \leq S^{0}-\min \left(S^{0}, \lambda_{1}\right)$ and $b_{0} \leq S^{0}-\min \left(S^{0}, \lambda_{2}\right)$.

Proof. We show that $\gamma_{0} \leq S^{0}-\min \left(S^{0}, \lambda_{1}\right)$. The proof for $b_{0} \leq S^{0}-\min \left(S^{0}, \lambda_{2}\right)$ is similar. We consider two cases.

Case 1. $\lambda_{1} \geq S^{0}$. It will suffice to show that $\delta_{0}=\gamma_{0}=0$. We first apply Theorem 3.1 to obtain $\lim _{t \rightarrow \infty} x_{1}(t)=0$. By Lemma 4.1 and the boundedness of $x_{1}(t)$, it follows that $\lim _{t \rightarrow \infty} x_{1}^{\prime}(t)=0$. Using the first equation of (4.5), we then have $\lim _{t \rightarrow \infty} y_{r_{1}}(t)=0$. Repeating this argument, we conclude that $\lim _{t \rightarrow \infty} y_{i}(t)=0$ for all $i=0,1,2, \ldots, r_{1}+1$. Now (4.6) gives $\lim _{t \rightarrow \infty} u_{0}(t)=0$, i.e., $\delta_{0}=\gamma_{0}=0$, as desired.

Case 2. $\lambda_{1}<S^{0}$. In this case, we apply Lemmas 4.1 and 4.2 to obtain a sequence $\left\{t_{m}\right\} \uparrow \infty$ such that

$$
\lim _{m \rightarrow \infty} u_{0}\left(t_{m}\right)=\gamma_{0} \quad \text { and } \quad \lim _{m \rightarrow \infty} u_{0}^{\prime}\left(t_{m}\right)=0 .
$$


From (4.7) it follows that

$$
\begin{aligned}
D \gamma_{0} & =\lim _{m \rightarrow \infty} D u_{0}\left(t_{m}\right) \\
& =\lim _{m \rightarrow \infty}\left[u_{r_{1}+1}\left(t_{m}\right) p_{1}\left(S^{0}-u_{0}\left(t_{m}\right)-v_{0}\left(t_{m}\right)+\rho\left(t_{m}\right)\right)\right] \\
& \leq \limsup _{m \rightarrow \infty} u_{r_{1}+1}\left(t_{m}\right) p_{1}\left(S^{0}-u_{0}\left(t_{m}\right)+\rho\left(t_{m}\right)\right) \\
& \leq \gamma_{r_{1}+1} p_{1}\left(S^{0}-\gamma_{0}\right) .
\end{aligned}
$$

Applying Lemma 4.3, we have from (4.14) that

$$
D \gamma_{0} \leq\left(\frac{\alpha_{1}}{D+\alpha_{1}}\right)^{r_{1}+1} \gamma_{0} p_{1}\left(S^{0}-\gamma_{0}\right)
$$

If $\gamma_{0}=0$, there is nothing to prove. If $\gamma_{0}>0,(4.15)$ implies that

$$
D \leq\left(\frac{\alpha_{1}}{D+\alpha_{1}}\right)^{r_{1}+1} p_{1}\left(S^{0}-\gamma_{0}\right)
$$

Therefore, by (2.4)

$$
S^{0}-\gamma_{0} \geq \lambda_{1} \text {, i.e., } \gamma_{0} \leq S^{0}-\lambda_{1},
$$

as desired.

LEMma 4.5. If $\lambda_{1}<\min \left(S^{0}, \lambda_{2}\right)$, then $\delta_{i}>0$ for all $i=0,1, \ldots, r_{1}+1$.

Proof. By (4.9), it suffices to show that $\delta_{0}>0$. First, we prove that

$$
\delta:=\liminf _{t \rightarrow \infty}\left[\alpha_{1} u_{0}(t)+\sum_{j=1}^{r_{1}+1} D\left(\frac{D+\alpha_{1}}{\alpha_{1}}\right)^{j-1} u_{j}(t)\right]>0 .
$$

Define

$$
w(t)=\alpha_{1} u_{0}(t)+\sum_{j=1}^{r_{1}+1} D\left(\frac{D+\alpha_{1}}{\alpha_{1}}\right)^{j-1} u_{j}(t), \quad t>0 .
$$

It follows from (4.7) that

$$
\begin{aligned}
w^{\prime}(t) & =-\frac{D\left(D+\alpha_{1}\right)^{r_{1}+1}}{\alpha_{1}^{r_{1}}} u_{r_{1}+1}(t)+\alpha_{1} p_{1}\left(S^{0}-u_{0}(t)-v_{0}(t)+\rho(t)\right) u_{r_{1}+1}(t) \\
& =-\alpha_{1} u_{r_{1}+1}(t)\left[D\left(\frac{D+\alpha_{1}}{\alpha_{1}}\right)^{r_{1}+1}-p_{1}\left(S^{0}-u_{0}(t)-v_{0}(t)+\rho(t)\right)\right] .
\end{aligned}
$$

Let $0<\varepsilon<\frac{1}{2}\left[\min \left(S^{0}, \lambda_{2}\right)-\lambda_{1}\right]$. We prove (4.16) by contradiction.

Suppose that $\delta=0$. Then there is a sequence $\left\{s_{m}\right\} \uparrow \infty$ such that for all $m$, $\rho\left(s_{m}\right)>-\frac{\varepsilon}{2}, w^{\prime}\left(s_{m}\right) \leq 0$, and $\lim _{m \rightarrow \infty} w\left(s_{m}\right)=0$. This implies that for all large $m, w\left(s_{m}\right)<\frac{1}{2} \alpha_{1} \varepsilon$. Notice that by $(4.17), \alpha_{1} u_{0}(t) \leq w(t)$. Thus $u_{0}\left(s_{m}\right)<\frac{1}{2} \varepsilon$ for all large $m$. Since $w^{\prime}\left(s_{m}\right) \leq 0$, it follows from (4.18) that

$$
\alpha_{1} u_{r_{1}+1}\left(s_{m}\right)\left[D\left(\frac{D+\alpha_{1}}{\alpha_{1}}\right)^{r_{1}+1}-p_{1}\left(S^{0}-u_{0}\left(s_{m}\right)-v_{0}\left(s_{m}\right)+\rho\left(s_{m}\right)\right)\right] \geq 0 .
$$


Since $u_{r_{1}+1}\left(s_{m}\right)>0$, the above inequality implies that

$$
S^{0}-u_{0}\left(s_{m}\right)-v_{0}\left(s_{m}\right)+\rho\left(s_{m}\right) \leq \lambda_{1} .
$$

Consequently, for all large $m$,

$$
\begin{aligned}
v_{0}\left(s_{m}\right) & \geq S^{0}-\lambda_{1}-u_{0}\left(s_{m}\right)+\rho\left(s_{m}\right) \\
& >S^{0}-\lambda_{1}-\varepsilon \\
& >S^{0}-\frac{\lambda_{1}}{2}-\frac{1}{2} \min \left(S^{0}, \lambda_{2}\right) \\
& =S^{0}-\min \left(S^{0}, \lambda_{2}\right)+\frac{1}{2}\left[\min \left(S^{0}, \lambda_{2}\right)-\lambda_{1}\right] .
\end{aligned}
$$

Thus $b_{0}=\lim \sup _{t \rightarrow \infty} v_{0}(t)>S^{0}-\min \left(S^{0}, \lambda_{2}\right)$, contradicting Lemma 4.4. Therefore, (4.16) holds and so $\delta>0$.

We now prove $\delta_{0}>0$. Suppose, to the contrary, that $\delta_{0}=0$. We then have a sequence $\left\{\tilde{s}_{m}\right\} \uparrow \infty$ such that $\lim _{m \rightarrow \infty} u_{0}\left(\tilde{s}_{m}\right)=0$. Note that from (4.6)

$$
u_{0}\left(\tilde{s}_{m}\right)=u_{r_{1}+1}\left(\tilde{s}_{m}\right)+\sum_{j=0}^{r_{1}} \frac{y_{j}\left(\tilde{s}_{m}\right)}{\alpha_{1}} .
$$

Since $y_{j}(t), j=0,1, \ldots, r_{1}$, are all positive for $t>0$, the above equation implies that

$$
\lim _{m \rightarrow \infty} u_{r_{1}+1}\left(\tilde{s}_{m}\right)=0 \quad \text { and } \quad \lim _{m \rightarrow \infty} y_{j}\left(\tilde{s}_{m}\right)=0, j=0,1,2, \ldots, r_{1} .
$$

Using (4.6) once more, we obtain $\lim _{m \rightarrow \infty} u_{j}\left(\tilde{s}_{m}\right)=0$ for all $j=0,1,2, \ldots, r_{1}+1$. Now (4.17) implies that $\lim _{m \rightarrow \infty} w\left(\tilde{s}_{m}\right)=0$, which leads to $\delta=0$, a contradiction. This proves $\delta_{0}>0$.

LEMma 4.6. If $\lambda_{1}<\lambda_{2}<S^{0}$, then $a_{j}=b_{j}=0$ for all $j=0,1,2, \ldots, r_{2}+1$.

Proof. By (4.10), it suffices to show that $a_{0}=b_{0}=0$. We first apply Lemmas 4.1 and 4.2 to the function $u_{0}(t)$ to obtain a sequence $\left\{s_{m}\right\} \uparrow \infty$ such that

$$
\lim _{m \rightarrow \infty} u_{0}\left(s_{m}\right)=\delta_{0} \text { and } \lim _{m \rightarrow \infty} u_{0}^{\prime}\left(s_{m}\right)=0 .
$$

It then follows from (4.7) that

$$
\lim _{m \rightarrow \infty} D u_{0}\left(s_{m}\right)=\lim _{m \rightarrow \infty} p_{1}\left(S^{0}-u_{0}\left(s_{m}\right)-v_{0}\left(s_{m}\right)+\rho\left(s_{m}\right)\right) u_{r_{1}+1}\left(s_{m}\right) .
$$

Let $\varepsilon>0$ be given. We have $\rho\left(s_{m}\right)>-\frac{\varepsilon}{2}, u_{r_{1}+1}\left(s_{m}\right) \geq \delta_{r_{1}+1}-\varepsilon$, and $v_{0}\left(s_{m}\right) \leq b_{0}+\frac{\varepsilon}{2}$ for all large $m$. Then (4.19) implies that

$$
\lim _{m \rightarrow \infty} D u_{0}\left(s_{m}\right) \geq \lim _{m \rightarrow \infty} p_{1}\left(S^{0}-u_{0}\left(s_{m}\right)-b_{0}-\varepsilon\right)\left(\delta_{r_{1}+1}-\varepsilon\right),
$$

or, equivalently,

$$
D \delta_{0} \geq p_{1}\left(S^{0}-\delta_{0}-b_{0}-\varepsilon\right)\left(\delta_{r_{1}+1}-\varepsilon\right) .
$$

Since $\varepsilon>0$ is arbitrary, upon letting $\varepsilon \rightarrow 0$, it follows that

$$
D \delta_{0} \geq p_{1}\left(S^{0}-\delta_{0}-b_{0}\right) \delta_{r_{1}+1} .
$$


By Lemma 4.3, the above inequality leads to

$$
D\left(\frac{D+\alpha_{1}}{\alpha_{1}}\right)^{r_{1}+1} \delta_{r_{1}+1} \geq p_{1}\left(S^{0}-\delta_{0}-b_{0}\right) \delta_{r_{1}+1} .
$$

Note that from Lemma $4.5, \delta_{r_{1}+1}>0$. Canceling $\delta_{r_{1}+1}$ in the above inequality yields

$$
S^{0}-\delta_{0}-b_{0} \leq \lambda_{1} .
$$

We now show that $b_{0}=0$. Suppose, to the contrary, that $b_{0}>0$. Let $\varepsilon>0$ be arbitrarily given. We apply Lemmas 4.1 and 4.2 once more to obtain a sequence $\left\{t_{m}\right\} \uparrow \infty$ such that $\rho\left(t_{m}\right)<\frac{\varepsilon}{2}$,

$$
\begin{aligned}
& \lim _{m \rightarrow \infty} v_{0}\left(t_{m}\right)=b_{0}, \quad \lim _{m \rightarrow \infty} v_{0}^{\prime}\left(t_{m}\right)=0, \text { and } \\
& v_{r_{2}+1}\left(t_{m}\right) \leq b_{r_{2}+1}+\varepsilon, \quad u_{0}\left(t_{m}\right) \geq \delta_{0}-\frac{\varepsilon}{2} .
\end{aligned}
$$

By (4.7), we have

$$
\begin{aligned}
D b_{0} & =\lim _{m \rightarrow \infty} D v_{0}\left(t_{m}\right) \\
& =\lim _{m \rightarrow \infty} p_{2}\left(S^{0}-u_{0}\left(t_{m}\right)-v_{0}\left(t_{m}\right)+\rho\left(t_{m}\right)\right) v_{r_{2}+1}\left(t_{m}\right) \\
& \leq \lim _{m \rightarrow \infty} p_{2}\left(S^{0}-\delta_{0}-v_{0}\left(t_{m}\right)+\varepsilon\right)\left(b_{r_{2}+1}+\varepsilon\right) \\
& =p_{2}\left(S^{0}-\delta_{0}-b_{0}+\varepsilon\right)\left(b_{r_{2}+1}+\varepsilon\right) .
\end{aligned}
$$

Letting $\varepsilon \rightarrow 0$, we obtain

$$
D b_{0} \leq p_{2}\left(S^{0}-\delta_{0}-b_{0}\right) b_{r_{2}+1} .
$$

Together with (4.10), this leads to

$$
D\left(\frac{D+\alpha_{2}}{\alpha_{2}}\right)^{r_{2}+1} b_{r_{2}+1} \leq p_{2}\left(S^{0}-\delta_{0}-b_{0}\right) b_{r_{2}+1} .
$$

Since $b_{0}>0$, by (4.21) $b_{r_{2}+1}>0$. Canceling $b_{r_{2}+1}$ in (4.22) yields

$$
S^{0}-\delta_{0}-b_{0} \geq \lambda_{2} \text {. }
$$

This contradicts (4.20) since, by assumption, $\lambda_{1}<\lambda_{2}$. Therefore, $b_{0}=0$ and hence $a_{0}=b_{0}=0$.

LEMMA 4.7. If $\delta_{0}>0$ and $a_{0}=b_{0}=0$, then $\delta_{i}=\gamma_{i}=\left(\frac{\alpha_{1}}{D+\alpha_{1}}\right)^{i}\left(S^{0}-\lambda_{1}\right)>0$ for all $i=0,1,2, \ldots, r_{1}+1$.

Proof. We first show that $\delta_{0}=\gamma_{0}$. Suppose for the purpose of contradiction that $\delta_{0}<\gamma_{0}$. By Lemma 4.2, there is a sequence $\left\{s_{m}\right\} \uparrow \infty$ such that

$$
\lim _{m \rightarrow \infty} u_{0}\left(s_{m}\right)=\delta_{0} \quad \text { and } \quad u_{0}^{\prime}\left(s_{m}\right)=0 \text { for all } m \text {. }
$$

By (4.7),

$$
D u_{0}\left(s_{m}\right)=u_{r_{1}+1}\left(s_{m}\right) p_{1}\left(S^{0}-u_{0}\left(s_{m}\right)-v_{0}\left(s_{m}\right)+\rho\left(s_{m}\right)\right) .
$$


Letting $m \rightarrow \infty$ on both sides of this equation and using (4.9) yield

$$
\begin{aligned}
D \delta_{0} & =\lim _{m \rightarrow \infty} u_{r_{1}+1}\left(s_{m}\right) p_{1}\left(S^{0}-\delta_{0}\right) \geq \delta_{r_{1}+1} p_{1}\left(S^{0}-\delta_{0}\right) \\
& \geq\left(\frac{\alpha_{1}}{D+\alpha_{1}}\right)^{r_{1}+1} \delta_{0} p_{1}\left(S^{0}-\delta_{0}\right) .
\end{aligned}
$$

Canceling $\delta_{0}>0$ in (4.23), we obtain $S^{0}-\delta_{0} \leq \lambda_{1}$; i.e., $\delta_{0} \geq S^{0}-\lambda_{1}$. Note that $\gamma_{0}>0$. By Lemma 4.4, we must have $\lambda_{1}<S^{0}$ and so $\gamma_{0} \leq S^{0}-\lambda_{1}$. This leads to $\delta_{0} \geq \gamma_{0}$, a contradiction. Therefore, $\delta_{0}=\gamma_{0}$.

We now show that $\delta_{0}=\gamma_{0}=S^{0}-\lambda_{1}$. Since $\lim _{t \rightarrow \infty} u_{0}(t)$ exists, (4.9) implies that $\lim _{t \rightarrow \infty} u_{i}(t)=\delta_{i}=\gamma_{i}$ exists for every $i=1,2, \ldots, r_{1}+1$. Note that by Lemma 4.1, $\lim _{t \rightarrow \infty} u_{0}^{\prime}(t)=0$. It follows from (4.7) that

$$
\lim _{t \rightarrow \infty}\left[-D u_{0}(t)+u_{r_{1}+1}(t) p_{1}\left(S^{0}-u_{0}(t)-v_{0}(t)+\rho(t)\right)\right]=0 .
$$

Together with (4.9), this gives

$$
D \delta_{0}=\delta_{r_{1}+1} p_{1}\left(S^{0}-\delta_{0}\right) \geq\left(\frac{\alpha_{1}}{D+\alpha_{1}}\right)^{r_{1}+1} \delta_{0} p_{1}\left(S^{0}-\delta_{0}\right) .
$$

Since $\delta_{0}>0$, canceling $\delta_{0}$ in (4.24) yields $S^{0}-\delta_{0} \leq \lambda_{1}$. By Lemma 4.4, this implies that $\delta_{0}=\gamma_{0}=S^{0}-\lambda_{1}$, as desired. The rest of the proof now follows immediately from (4.9).

We are now in the position to prove Theorems 3.3 and 3.4 .

Proof of Theorem 3.3. Without loss of generality, we assume $\lambda_{i}=\lambda_{1}$ and $\lambda_{j}=$ $\lambda_{2}$. We first apply Lemmas 4.4 and 4.3 to conclude that $a_{j}=b_{j}=0$ for all $j=$ $0,1, \ldots, r_{2}+1$. Note that by Lemma $4.5, \delta_{0}>0$. It now follows from Lemma 4.7 that $\delta_{0}=\gamma_{0}=S^{0}-\lambda_{1}$ and

$$
\delta_{r_{1}+1}=\gamma_{r_{1}+1}=\left(\frac{\alpha_{1}}{D+\alpha_{1}}\right)^{r_{1}+1}\left(S^{0}-\lambda_{1}\right) .
$$

Consequently, by (2.8),

$$
\begin{aligned}
\lim _{m \rightarrow \infty}\left(S^{0}-S(t)+\rho(t)\right) & =\lim _{t \rightarrow \infty}\left[\sum_{i=0}^{r_{1}} \frac{y_{i}(t)}{\alpha_{1}}+\sum_{j=0}^{r_{2}} \frac{z_{j}(t)}{\alpha_{2}}+x_{1}(t)+x_{2}(t)\right] \\
& =\lim _{t \rightarrow \infty}\left[u_{0}(t)+v_{0}(t)\right] \\
& =\gamma_{0}+b_{0}=S^{0}-\lambda_{1} .
\end{aligned}
$$

Hence, $\lim _{t \rightarrow \infty} S(t)=\lambda_{1}$. This leads to

$$
\begin{aligned}
\lim _{t \rightarrow \infty} \pi(\phi ; t) & =\lim _{t \rightarrow \infty}\left(S(t), u_{r_{1}+1}(t), v_{r_{2}+1}(t)\right) \\
& =\left(\lambda_{1}, \gamma_{r_{1}+1}, b_{r_{2}+1}\right) \\
& =\left(\lambda_{1},\left(\frac{\alpha_{1}}{D+\alpha_{1}}\right)^{r_{1}+1}\left(S^{0}-\lambda_{1}\right), 0\right)=E_{1},
\end{aligned}
$$

as required. 
TABLE 1

Basic run parameters.

\begin{tabular}{cccccc}
\hline Strain & $\begin{array}{c}c_{i} \\
\text { cell } / \mathrm{g}\end{array}$ & $\begin{array}{c}k_{i} \\
\mathrm{~g} / \text { liter }\end{array}$ & $\begin{array}{c}m_{i} \\
\text { per hr}\end{array}$ & $\begin{array}{c}N_{i}(0) \\
\text { cell/liter }\end{array}$ & $\begin{array}{c}D=\frac{F}{V} \\
\text { per hr }\end{array}$ \\
\hline$N_{1}$ & $6.3 \times 10^{10}$ & $1.6 \times 10^{-6}$ & 0.68 & $10^{4.25}$ & $7.5 \times 10^{-2}$ \\
$N_{2}$ & $6.2 \times 10^{10}$ & $1.6 \times 10^{-6}$ & 0.96 & $10^{3.1}$ & \\
\hline
\end{tabular}

\begin{tabular}{ccc}
\hline Figure & $\begin{array}{c}S(0) \\
\mathrm{g} / \text { liter }\end{array}$ & $\begin{array}{c}S^{0} \\
\mathrm{~g} / \text { liter }\end{array}$ \\
\hline 1,2 & $2 \times 10^{-6}$ & $5 \times 10^{-6}$ \\
3 & $2 \times 10^{-7}$ & $2 \times 10^{-7}$ \\
\hline
\end{tabular}

Proof of Theorem 3.4. Assume that $\lambda_{i}=\lambda_{1}, \lambda_{j}=\lambda_{2}$; i.e., $\lambda_{1}<\lambda_{2}<S^{0}$. We first apply Lemmas 4.5 and 4.6 to obtain $\delta_{0}>0$ and $a_{j}=b_{j}=0$ for all $j=0,1, \ldots, r_{2}+1$. By Lemma 4.7, it follows that $\delta_{0}=\gamma_{0}=S^{0}-\lambda_{1}$ and

$$
\delta_{r_{1}+1}=\gamma_{r_{1}+1}=\left(\frac{\alpha_{1}}{D+\alpha_{1}}\right)^{r_{1}+1}\left(S^{0}-\lambda_{1}\right) .
$$

The rest of the proof is the same as that for Theorem 3.3.

5. Observations and numerical simulations. In this section we make some numerical observations, based on computer simulations using Matlab, about the possible effects of including delay in the conversion process in the basic chemostat model. In order for the simulations to be somewhat biologically meaningful, at least with respect to orders of magnitude, we used the data given for the first two experiments done in Hansen and Hubbell [20]. The outcome of all of the simulations agreed with the theoretical predictions in this paper. In order to use the data given in [20], we simulated model (1.1) and model (2.6) without the yield constants scaled out. That is, throughout $(2.6), x_{i}(t)$ was replaced by $N_{i}(t)=c_{i} x_{i}(t), y_{i}(t)$ was replaced by $c_{i} y_{i}(t)$, and $z_{i}(t)$ was replaced by $c_{i} z_{i}(t)$. The equilibrium concentration of the surviving population is therefore given by $N_{i}^{*}=c_{i}\left(S^{0}-\lambda_{i}\right)$. All of the simulations done on model (2.6) represent solutions of model (2.1) for some choice of initial data.

We noticed some interesting characteristics of the solutions that were consistent in all of the simulations. We describe these observations below and illustrate them using the data from the second experiment done in Hansen and Hubbell for two strains of Escherichia coli. In order to focus on certain aspects of the solutions, the graphs are only shown for 150 hours. However, many of the simulations were done for 2000 hours at a tolerance of $10^{-12}$ to make sure that the convergence was as predicted.

Hansen and Hubbell [20] used the Michaelis-Menten form to model the response functions $p_{i}(S)=\frac{m_{i} S}{k_{i}+S}$. The basic run parameters for all of the simulations, based on experiment 2 in [20], are summarized in Table 1.

The initial conditions, $N_{i}(0)$, were estimated from a graph given in Hansen and Hubbell. However, there was no indication of what $S(0)$ was in their paper, so this was selected arbitrarily. In Figure 3, in order to emphasize the point we wished to make, we were required to select a different value for $S^{0}$. Since it was not possible to know the past history of the microorganisms used in the experiment of Hansen and Hubbell we chose the initial data (for $t<0$ ) arbitrarily. Figures 1 (a), (c) and 2 (a), (c), (e) were done using one choice for the initial data and Figures 1 (b), (d) and 3 (b), (d), (f) were done using another. 
We first make some general observations. Note that model (2.1) and hence model (2.6) approach the discrete delay model (1.2) as a limiting case (see Appendix). The criteria for the prediction of the outcome given in this paper can approximate the criteria necessary for the outcome in the discrete delay case. To be more specific, let us denote by $\lambda_{i}\left(\tau_{i}\right)$ the corresponding $\lambda$ values for the discrete delay model (1.2). For convenience, we assume that $p_{1}$ and $p_{2}$ in (1.2) and (2.1) are locally Lipschitz and strictly increasing with $p_{1}(0)=p_{2}(0)=0$. This implies that, for each $i \in\{1,2\}$,

$$
\lambda_{i}\left(\tau_{i}\right)=p_{i}^{-1}\left(D e^{D \tau_{i}}\right),
$$

$$
\lambda_{i}\left(\alpha_{i}, r_{i}\right)=p_{i}^{-1}\left(D\left(\frac{D+\alpha_{i}}{\alpha_{i}}\right)^{r_{i}+1}\right) .
$$

As in [32], if we let $r_{i}$ go to infinity while keeping the mean delay, $\frac{r_{i}+1}{\alpha_{i}}=\tau_{i}$ fixed, it follows from (5.1) that

$$
\begin{aligned}
\lim _{r_{i} \rightarrow \infty} \lambda_{i}\left(\alpha_{i}, r_{i}\right) & =\lim _{r_{i} \rightarrow \infty} p_{i}^{-1}\left(D\left(\frac{D+\alpha_{i}}{\alpha_{i}}\right)^{r_{i}+1}\right) \\
& =p_{i}^{-1}\left(\lim _{\alpha_{i} \rightarrow \infty} D\left(1+\frac{D}{\alpha_{i}}\right)^{\alpha_{i} \tau_{i}}\right) \\
& =p_{i}^{-1}\left(D e^{D \tau_{i}}\right)=\lambda_{i}\left(\tau_{i}\right) .
\end{aligned}
$$

This observation was made by MacDonald [32], as far as the local stability analysis was concerned. The theoretical results in this paper demonstrate the global significance of this observation. The numerical simulations (see Figures 1 and 2) seem to show that it is the mean delay, rather than the order of the kernel that plays the most important role.

On the other hand, the ODEs model (1.1) can also be thought of as a limiting case of the distributed delay model and it can be shown that if the mean delays in the distributed delay model (2.1) are sufficiently small, then the predictions about whether a population survives or dies out will be the same as the predictions of the ODEs model. To see this note that if $\tau_{i}=0$ in model (1.2), the discrete delay model becomes the ODEs model. Therefore, $\lambda_{i}(0)$ can also be used to denote the breakeven concentrations in the ODEs model. Since each $\lambda_{i}\left(\alpha_{i}, r_{i}\right)$ is in fact a continuous function of $\alpha_{i}$ and the kernel order $r_{i}$ (see (5.1)), if $\lambda_{1}(0)<\lambda_{2}(0)$ it follows that if the mean delay $\tau_{i}$ is sufficiently small (e.g., let $\alpha_{i} \rightarrow \infty$ with $\tau_{i} \rightarrow 0$ ), then $\lambda_{1}\left(\alpha_{1}, r_{1}\right)<$ $\lambda_{2}\left(\alpha_{2}, r_{2}\right)$ and, also, the relative values of the $\lambda_{i}$ 's and $S^{0}$ will be maintained. Hence it seems, at least qualitatively, that small mean delays can be neglected.

We now describe the numerical simulations. In Figures 1-3, to distinguish $N_{1}(t)$ and $N_{2}(t)$, use the relative values of $N_{1}(0)$ and $N_{2}(0)$ given in Table 1. The first figure demonstrates the effect of varying the members of the delay kernel (i.e., varying the $r_{i}$ and $\alpha_{i}$ ) while holding the mean delays (i.e., $\tau_{i}=\frac{r_{i}+1}{\alpha_{i}}$ ) fixed. In Figures 1 (a) and (b) we show the effect of increasing the order of the kernel for both populations while keeping the mean delay fixed. Although there is a small difference between the case of $r_{1}=r_{2}=0$ and $r_{1}=r_{2}=20$, for the scale of the graph the cases of $r_{1}=r_{2}=20$, $r_{1}=r_{2}=40$, and $r_{1}=r_{2}=60$ are indistinguishable. In Figures 1 (c) and (d) we show the effect of increasing the order of one of the kernels. For the initial data used for Figure 1 (c), there was very little difference among all of the cases. However, for Figure $1(\mathrm{~d})$, the difference between $r_{1}=40$ and $r_{2}=0$ and the other cases during 
(a)

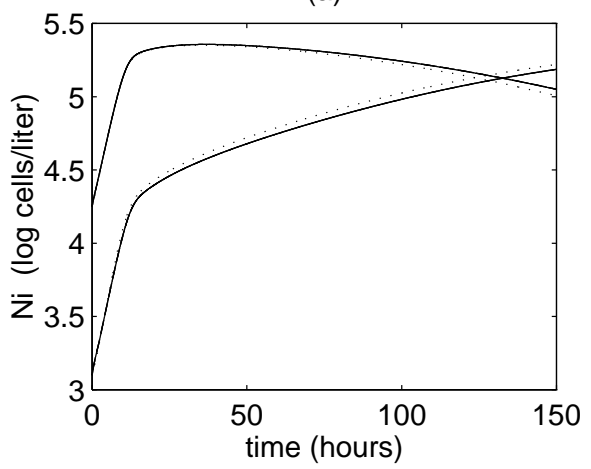

(c)

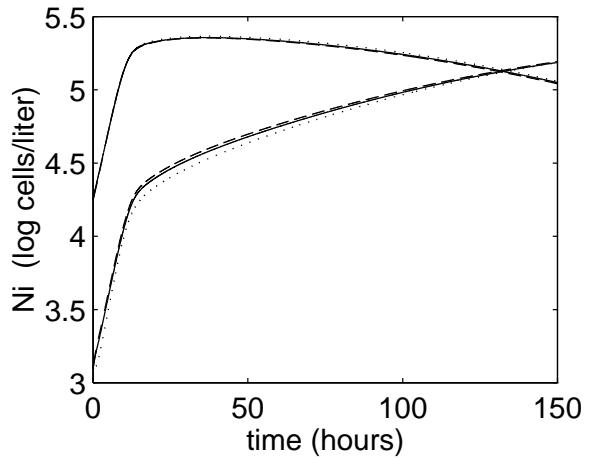

(b)

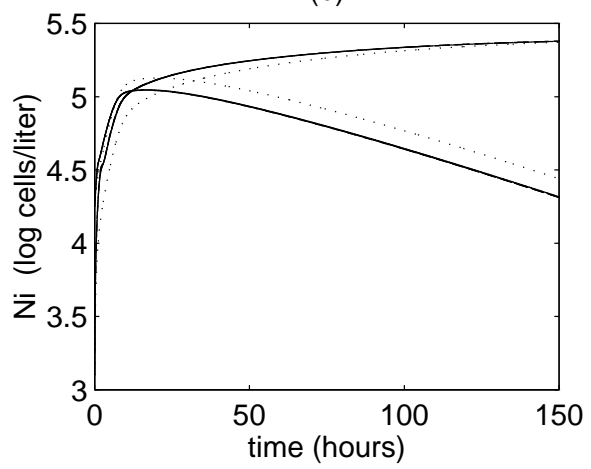

(d)

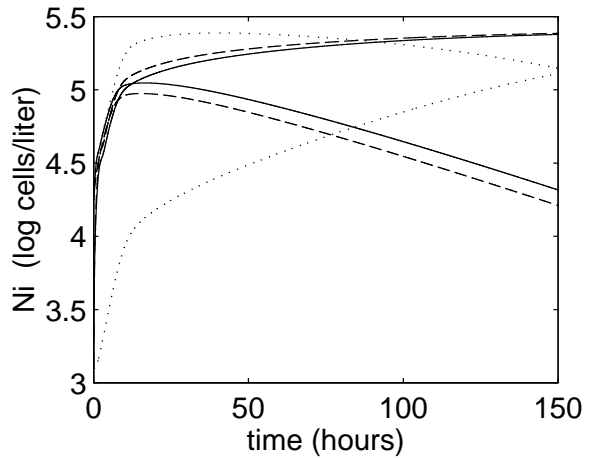

FIG. 1. The effect of varying $r_{i}$ and $\alpha_{i}$ while holding $\tau_{i}=\frac{r_{i}+1}{\alpha_{i}}$ fixed. Figures 1 (a) and (c) were done using one set of initial data and Figures $1(\mathrm{~b})$ and $(\mathrm{d})$ were done using another. The key to this figure is summarized in Tables 1 and 2.

TABLE 2

Run information and key for Figure 1.

\begin{tabular}{ccccccccc}
\hline Strain & $\begin{array}{c}\tau_{i} \\
\text { hours }\end{array}$ & $r_{i}$ & $\begin{array}{c}\alpha_{i} \\
\text { per } \mathrm{hr}\end{array}$ & $\begin{array}{c}\lambda_{i} \\
\mathrm{~g} / \mathrm{liter}\end{array}$ & $\begin{array}{c}N_{i}^{*} \\
\text { cells/liter }\end{array}$ & $\begin{array}{c}N_{i}^{*} \\
\log _{10} \text { cells/liter }\end{array}$ & Figure & $\begin{array}{c}\text { Line } \\
\text { type }\end{array}$ \\
\hline$N_{1}$ & 1 & 0 & 1 & $2.15 \times 10^{-7}$ & 0 & $-\infty$ & $1(\mathrm{a}),(\mathrm{b})$ & $\cdots$ \\
$N_{2}$ & 2 & 0 & 0.5 & $1.58 \times 10^{-7}$ & 261050 & 5.417 & & \\
\hline$N_{1}$ & 1 & 20 & 21 & $2.16 \times 10^{-7}$ & 0 & $-\infty$ & $1(\mathrm{a})-(\mathrm{d})$ & - \\
$N_{2}$ & 2 & 20 & 10.5 & $1.60 \times 10^{-7}$ & 258439 & 5.412 & & \\
\hline$N_{1}$ & 1 & 40 & 41 & $2.16 \times 10^{-7}$ & 0 & $-\infty$ & $1(\mathrm{a}),(\mathrm{b})$ & --- \\
$N_{2}$ & 2 & 40 & 20.5 & $1.60 \times 10^{-7}$ & 258369 & 5.142 & & \\
\hline$N_{1}$ & 1 & 60 & 61 & $2.16 \times 10^{-7}$ & 0 & $-\infty$ & $1(\mathrm{a}),(\mathrm{b})$ & $-\cdot-\cdot$ \\
$N_{2}$ & 2 & 60 & 30.5 & $1.60 \times 10^{-7}$ & 258345 & 5.147 & & \\
\hline$N_{1}$ & 1 & 40 & 41 & $2.16 \times 10^{-7}$ & 0 & $-\infty$ & $1(\mathrm{c}),(\mathrm{d})$ & $\cdots$ \\
$N_{2}$ & 2 & 0 & 0.5 & $1.58 \times 10^{-7}$ & 261050 & 5.142 & & \\
\hline$N_{1}$ & 1 & 0 & 1 & $2.15 \times 10^{-7}$ & 0 & $-\infty$ & $1(\mathrm{c}),(\mathrm{d})$ & --- \\
$N_{2}$ & 2 & 40 & 20.5 & $1.60 \times 10^{-7}$ & 258369 & 5.142 & & \\
\hline
\end{tabular}

the first 150 hours was very noticeable, although asymptotically the result was almost identical, as seen by the values of $N_{i}^{*}$ and $\lambda_{i}$. Although not depicted, the graphs for the cases of $r_{1}=60$ and $r_{2}=0$ as well as $r_{1}=80$ and $r_{2}=0$ were indistinguishable from the case of $r_{1}=40$ and $r_{2}=0$. Thus, Figure 1 seems to demonstrate that the order of the kernel does not play a very significant role and that an order of 40 may be a good approximation for the discrete delay case. Also, Figure 1 seems to show 
(a)

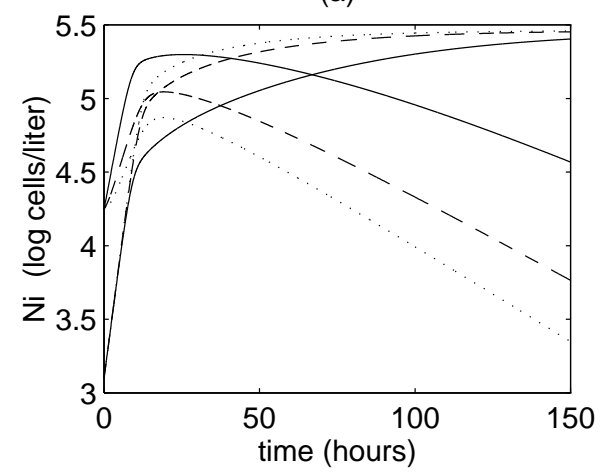

(c)

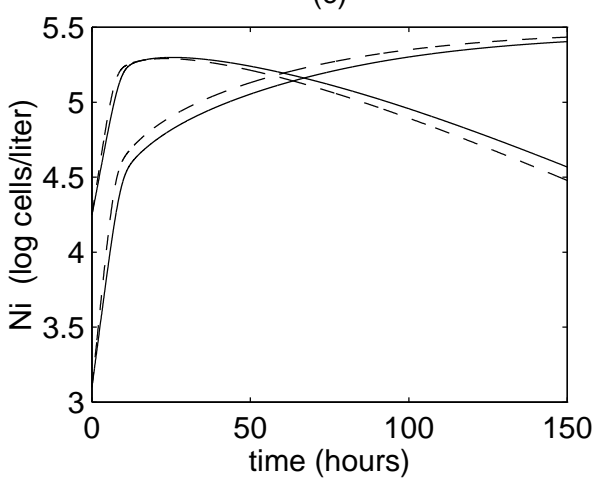

(e)

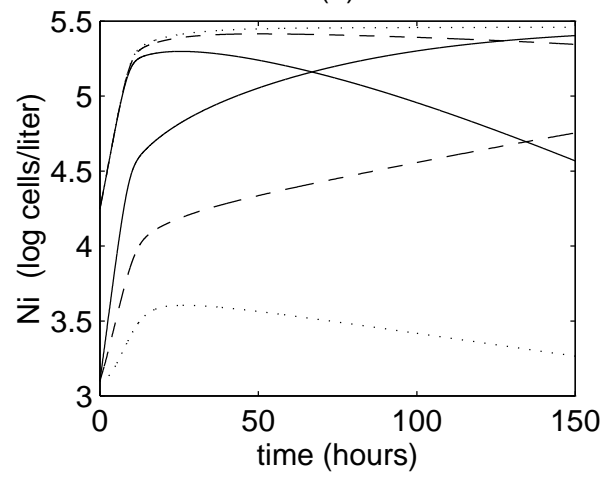

(b)

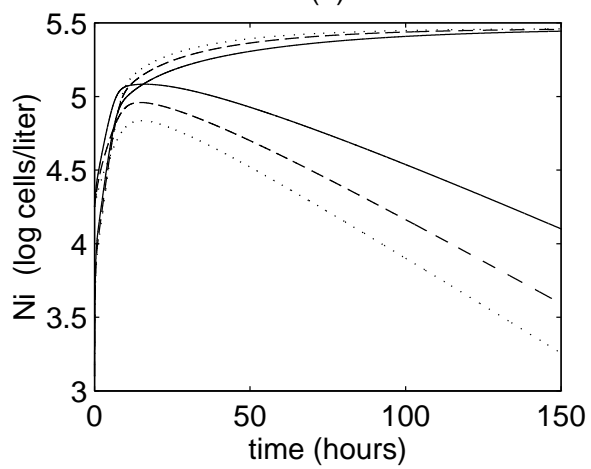

(d)

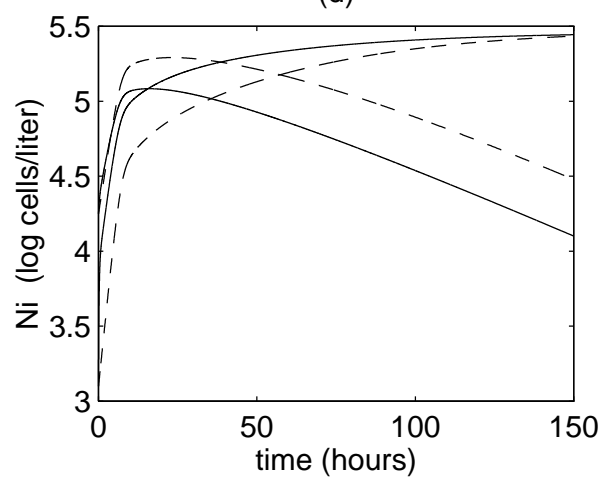

(f)

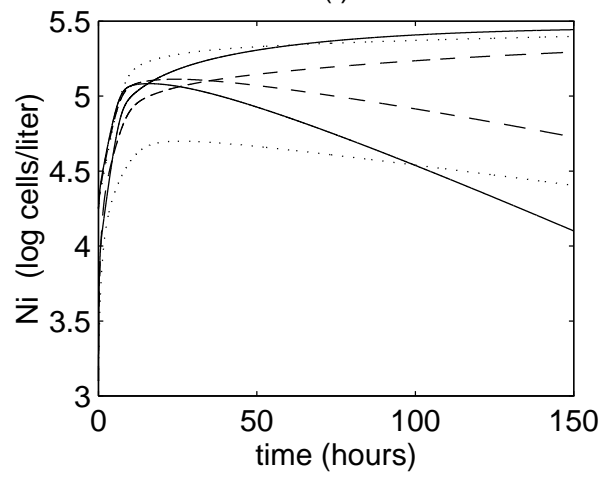

FIG. 2. The effect of varying $\tau_{i}$ while holding $r_{i}$ fixed. Figures 2 (a), (c), and (e) were done using one set of initial data, and Figures $2(\mathrm{~b}),(\mathrm{d})$, and (f) were done using another. The key to this figure is summarized in Tables 1 and 3.

that although the initial data plays no role in determining the asymptotic outcome, it can affect the initial transients and hence how long it takes for the culture to reach near equilibrium concentrations. Figure 1 (d) indicates that the order of the kernel could similarly affect the initial transients, although as shown by Figure 1 (a), (b), and (c), this need not generally be the case.

The second figure demonstrates the effect of varying the mean delay $\tau_{i}$ while holding the order of the kernels $r_{i}$ fixed. In order to make comparisons, the simulation with $\tau_{1}=0.5=\tau_{2}$ appears in all of the graphs in Figure 2. In Figures 2 (c) and (d) this simulation is compared with the simulation of the ODEs model. For the initial 
TABLE 3

Run information and key for Figure 2.

\begin{tabular}{ccccccccc}
\hline Strain & $\begin{array}{c}\tau_{i} \\
\text { hours }\end{array}$ & $r_{i}$ & $\begin{array}{c}\alpha_{i} \\
\text { per hr }\end{array}$ & $\begin{array}{c}\lambda_{i} \\
\mathrm{~g} / \text { liter }\end{array}$ & $\begin{array}{c}N_{i}^{*} \\
\text { cells/liter }\end{array}$ & $\begin{array}{c}N_{i}^{*} \\
\log _{10} \text { cells/liter }\end{array}$ & Figure & $\begin{array}{c}\text { Line } \\
\text { type }\end{array}$ \\
\hline$N_{1}$ & 0 & & ODE & $1.98 \times 10^{-7}$ & 0 & $-\infty$ & $2(\mathrm{c}),(\mathrm{d})$ & --- \\
$N_{2}$ & 0 & & ODE & $1.35 \times 10^{-7}$ & 301593 & 5.479 & & \\
\hline$N_{1}$ & 5 & 0 & 0.2 & $2.86 \times 10^{-7}$ & 0 & $-\infty$ & $2(\mathrm{a}),(\mathrm{b})$ & $\cdots$ \\
$N_{2}$ & 0.5 & 1 & 4 & $1.41 \times 10^{-7}$ & 290260 & 5.463 & & \\
\hline$N_{1}$ & 3 & 0 & 0.33 & $2.50 \times 10^{-7}$ & 0 & $-\infty$ & $2(\mathrm{a}),(\mathrm{b})$ & --- \\
$N_{2}$ & 0.5 & 1 & 4 & $1.41 \times 10^{-7}$ & 290260 & 5.463 & & \\
\hline$N_{1}$ & 0.5 & 0 & 2 & $2.07 \times 10^{-7}$ & 0 & $-\infty$ & $2(\mathrm{a})-(\mathrm{f})$ & - \\
$N_{2}$ & 0.5 & 1 & 4 & $1.41 \times 10^{-7}$ & 290260 & 5.463 & & \\
\hline$N_{1}$ & 0.5 & 0 & 2 & $2.07 \times 10^{-7}$ & 0 & $-\infty$ & $2(\mathrm{e}),(\mathrm{f})$ & --- \\
$N_{2}$ & 3 & 1 & 0.67 & $1.71 \times 10^{-7}$ & 241894 & 5.384 & & \\
\hline$N_{1}$ & 0.5 & 0 & 2 & $2.07 \times 10^{-7}$ & 291060 & 5.464 & $2(\mathrm{e}),(\mathrm{f})$ & $\cdots$ \\
$N_{2}$ & 8 & 1 & 0.25 & $2.43 \times 10^{-7}$ & 0 & $-\infty$ & & \\
\hline
\end{tabular}

data in Figure 2(c), the delay of $\frac{1}{2}$ hour slowed down the convergence to equilibrium concentrations a small amount, whereas for the initial data in Figure 2 (d), the delay increased the rate of convergence.

Figures 2 (a) and (b) illustrate the effect of increasing the mean delay of the losing strain for the ODEs model from $\frac{1}{2}$ hour to 3 hours to 5 hours, while keeping the mean delay of the survivor for the ODEs model fixed at $\frac{1}{2}$ hour. In all of the simulations, the increase in the delay for the losing strain sped up the death rate of this strain. In the experiment by Hansen and Hubbell, they observed that the actual death rate was more rapid than the rate predicted by the ODEs model. Our simulations indicate that this could be accounted for if the mean delay of the losing strain was larger than that of the surviving strain.

On the other hand, Figures 2 (e) and (f) show the effect of increasing the mean delay of the surviving strain according to the predictions of the ODEs model from $\frac{1}{2}$ hour to 3 hours to 8 hours while keeping the mean delay of the losing strain according to the predictions of the ODEs model fixed at $\frac{1}{2}$ hour. The 3 -hour delay for the surviving strain significantly slowed down the death rate of the losing strain, and the 8-hour delay resulted in a reversal in prediction of the outcome of the competition; that is, the losing competitor for the ODEs model survived and the surviving competitor for the ODEs model approached extinction. This seems to be consistent with some experimental evidence. Tilman [45] reported experiments showing the dependence of the outcome of competition on temperature, and Halbach [19] did experiments that seem to indicate that the length of the time delay in growth response in laboratory populations is a function of controlled temperature. See also Herbert [22] for a discussion of the effect of temperature on substrate uptake of microorganisms.

Figure 3 demonstrates that including delay in the model can account for more oscillatory behavior in the initial transients, compared to the ODEs model. However, to obtain this behavior, we had to reduce $S^{0}$ from the value used in the actual experiment in [20]. Perhaps making a better selection for the initial data could have had a similar effect, but although it seems clear that the initial data affect the transients, it is still unclear how to manipulate the transients by means of the initial data.

We make some final remarks concerning the numerical simulations. Figures 1 and 2 demonstrate that the selection of the initial data does affect the transient behavior. However, it is usually, if not always, impossible to know the life history of the 


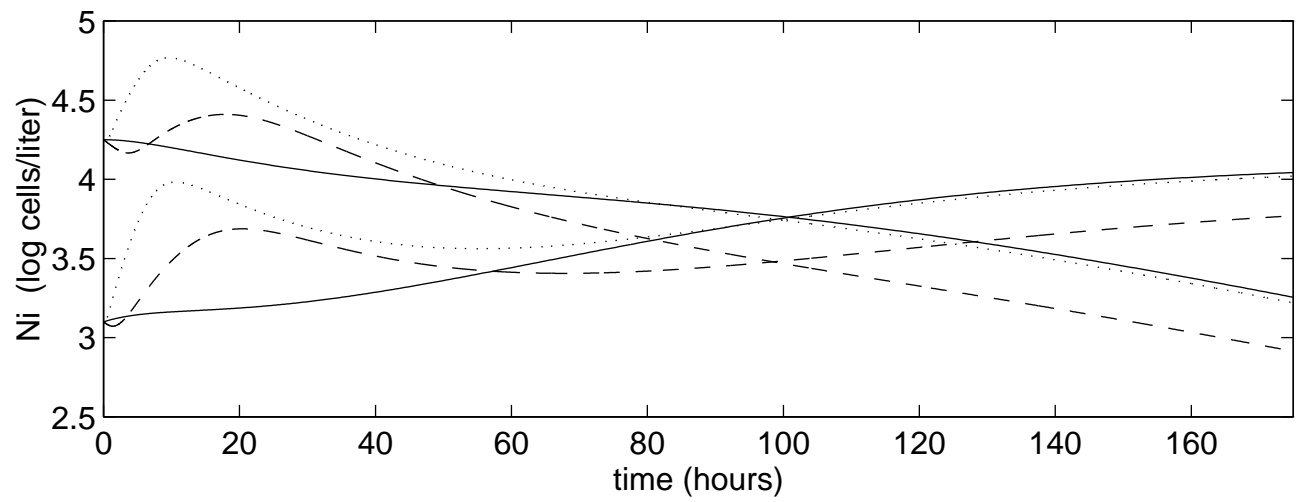

FIG. 3. An example in which delay accounts for more oscillation in the transients. The key to this figure is summarized in Tables 1 and 4.

TABLE 4

Run information and key for Figure 3.

\begin{tabular}{cccccccc}
\hline Strain & $\begin{array}{c}\tau_{i} \\
\text { hours }\end{array}$ & $r_{i}$ & $\begin{array}{c}\alpha_{i} \\
\text { per hr }\end{array}$ & $\begin{array}{c}\lambda_{i} \\
\text { g/liter }\end{array}$ & $\begin{array}{c}N_{i}^{*} \\
\text { cells/liter }\end{array}$ & $\begin{array}{c}N_{i}^{*} \\
\log _{10} \text { cells/liter }\end{array}$ & $\begin{array}{c}\text { Line } \\
\text { type }\end{array}$ \\
\hline$N_{1}$ & 0 & & ODE & $1.98 \times 10^{-7}$ & 0 & $-\infty$ & - \\
$N_{2}$ & 0 & & ODE & $1.36 \times 10^{-7}$ & 13293 & 4.124 & \\
\hline$N_{1}$ & 0.6 & 3 & 6.67 & $2.09 \times 10^{-7}$ & 0 & $-\infty$ & $\cdots$ \\
$N_{2}$ & 0.4 & 1 & 5 & $1.40 \times 10^{-7}$ & 12635 & 4.102 & $-\cdots$ \\
\hline$N_{1}$ & 6 & 3 & 0.67 & $3.25 \times 10^{-7}$ & 0 & $-\infty$ & 3.890 \\
$N_{2}$ & 4 & 1 & 0.5 & $1.84 \times 10^{-7}$ & 7765 & \\
\hline
\end{tabular}

microorganisms. Therefore it seems unlikely that this approach to modeling will ever yield a good predictor of the exact initial transient behavior for a specific experiment. However, Figures 1-3 seem to indicate that it could well be delay that accounts for some of the discrepancies that have been observed between experiments and the predictions of the ODEs model, and that delay seems to have certain consistent effects that can be used to enhance predictions, or to obtain information about the relative length of the delays in the conversion process for two different populations.

6. Discussion. In this paper, we studied a class of two species competition models in a chemostat. We included distributed delay to model the lag between the consumption of nutrient and the change in the concentration of the consuming populations. By using the linear chain trick technique and the fluctuation lemma, we determined the global asymptotic behavior.

The predictions based on the model involving distributed delay are similar to those for the corresponding chemostat model with discrete delay (see Ellermeyer [15], Hsu, Waltman, and Ellermeyer [26], and Wolkowicz and Xia [48]) and with no delay (see Hsu, Hubbell, and Waltman [25], Hsu [24], Butler and Wolkowicz [7], and Wolkowicz and $\mathrm{Lu}[47])$. In all of these models, all species concentrations, eventually approach equilibrium concentrations, and hence no nontrivial periodic solutions are possible. However, it must be remembered that the critical parameters $\lambda_{1}$ and $\lambda_{2}$ depend on the delay kernel chosen and so including delays in the model can change the relative values of the $\lambda$ 's and hence the predictions about whether a population survives or dies out. Also, as proved in the appendix, the discrete delay model (1.2) and the ODEs model (1.1) can be thought of as limiting cases of the distributed delay model. 
Our predictions can be summarized as follows. If $\lambda_{i} \geq S^{0}$ for some $i \in\{1,2\}$, then the chemostat environment cannot support population $x_{i}$ and it will be washed out, regardless of whether or not there is a competitor. This is an example of competitionindependent extinction. If $\lambda_{i}<S^{0} \leq \lambda_{j}$, then population $x_{i}$ survives and $x_{j}$ is washed out. Finally, if $\lambda_{i}<\lambda_{j}<S^{0}$, then again population $x_{i}$ survives and the competition eliminates the competitor $x_{j}$ (with the larger $\lambda$ value); i.e., competitive exclusion occurs. Note that in this last case, each species can survive in the chemostat in the absence of the other, and thus competition is the agent of elimination.

In a recent paper, Wolkowicz and Xia [48] discussed discrete delay effects on the predicted outcomes of competition. As in the case of the discrete delay model (1.2), distributed delay can also alter the predicted outcomes of competition. To be more precise, since $\lambda_{i}$ is a continuous function of $\alpha_{i}$ and the kernel order $r_{i}$, if we increase the mean delay $\tau_{i}=\frac{r_{i}+1}{\alpha_{i}}$, it can be seen from (5.1) that the corresponding $\lambda_{i}$ will also increase. This, together with our results, implies that a species with smaller $\lambda(0)$ value may lose the competition if its actual mean delay is sufficiently large (see Figures 2 (e) and (f)). As a consequence, compared with the ODEs model, the distributed delay model (2.1) may give completely different predictions about whether or not a population survives.

Cooke and Grossman [10] point out that two models with parallel structure, one with discrete delay and one with distributed delay, may not exhibit the same qualitative behavior (see MacDonald [33] for a general discussion of the effects of incorporating distributed delay in biological models). In theory, it is also possible for the predictions based on model (2.1) to differ from those given by the discrete delay model (1.2), since it is possible to arrange $\alpha_{i}$ and $r_{i}$ with $\tau_{i}=\frac{r_{1}+1}{\alpha_{i}}$ in such a way that inequalities

$$
\lambda_{2}\left(\alpha_{2}, r_{2}\right)<\lambda_{1}\left(\alpha_{1}, r_{1}\right)<\lambda_{1}\left(\tau_{1}\right)<\lambda_{2}\left(\tau_{2}\right)<S^{0}
$$

hold. Thus model (2.1) would predict population $x_{2}$ as the winner but model (1.2) would predict population $x_{1}$ as the winner. However, the numerical simulations seem to show that this inconsistency in predictions may not be likely in the chemostat. For example (see Table 2), using the data from the second experiment of Hansen and Hubbell, increasing $r_{i}$ from 0 to 20 changed $\lambda_{i}$ by at most 0.002 micrograms per liter, and further increases seemed to have even less effect. Hansen and Hubbell considered the $\lambda_{i}$ 's equal if they differed by 0.001 micrograms per liter as indicated by their third experiment, and simulations done on populations where $\left|\lambda_{1}-\lambda_{2}\right| \approx 0.002$ micrograms had to be run beyond 2000 hours to determine which population had the advantage and beyond 3000 hours to eliminate the possibility of coexistence. The order of magnitude of changes in the $\lambda_{i}$ 's due to changes in the mean delay are much more significant (see Table 3).

The results in this paper depend on the choice of the family of delay kernels (2.2), since this choice makes it possible to apply the linear chain trick technique. This form of distributed delay kernels has been widely used in biological modeling (see Cushing [12], MacDonald [32] and the references therein) and seems to be the most useful family of reducible kernels (i.e., delay kernels that allow a distributed delay model to be converted to an equivalent system of ordinary differential equations). Not only are these kernels mathematically convenient, but also linear combinations of them represent a generic class of distributed delay kernels (see Busenberg and Travis [5]). As far as the local asymptotic stability is concerned, in [5], they showed that there always exists an appropriate reducible system that can be analyzed instead of 
the actual (not necessarily reducible) delay differential equations system. However, empirical evidence (see, for example, Caperon [8]) seems to suggest that the nonreducible distribution should be used in parallel with the gamma distribution (2.2). In order to show the robustness of the global results we obtained, it is of practical importance to investigate the question of whether or not similar results hold for more general delay kernels. Unfortunately, the technique used in this paper cannot be used for general delay kernels, although it is possible to apply it in principle to linear combinations of members of the family of kernels (2.2).

It is not usually possible to know the past history of the microorganisms or the actual form of the delay kernel. Also, a particular member of the family of kernels (2.2) is at best an approximation. Therefore, it is not likely that $\alpha_{i}$ and $r_{i}$ have any real biological meaning. However, the quantitative as well as qualitative dynamic behavior of the model depends on the parameters $\alpha_{i}$ and $r_{i}$ in the delay kernels. As our computer simulations seem to indicate, it is the mean delay $\tau_{i}=\frac{r_{i}+1}{\alpha_{1}}$ rather than the order of the kernel that plays the more significant role. It might be possible to get some idea of the length of the mean delay from experiments. Assume that the dilution rate $D$ and the nutrient input concentration $S^{0}$ are held constant and we culture the species $N_{i}$ alone in the chemostat. If a positive steady-state equilibrium is reached in the culture, we measure the yield constant $c_{i}$ and the equilibrium concentrations of the surviving species $N_{i}$ and of the nutrient $S$, that is, $N_{i}^{*}$ and $\lambda_{i}$, respectively. Then, by our theory, we obtain the equilibrium equation

$$
N_{i}^{*}=c_{i}\left(\frac{\alpha_{i}}{D+\alpha_{i}}\right)^{r_{i}+1}\left(S^{0}-\lambda_{i}\right)
$$

This implies that the mean delays

$$
\tau_{i}=\frac{\ln \frac{c_{i}\left(S^{0}-\lambda_{i}\right)}{N_{i}^{*}}}{\ln \left(1+\frac{D}{\alpha_{i}}\right)^{\alpha_{i}}} \approx \frac{\ln \frac{c_{i}\left(S^{0}-\lambda_{i}\right)}{N_{i}^{*}}}{D}
$$

when the $\alpha_{i}$ are large. Thus, although different choices for $\alpha_{i}$ and $r_{i}$ may satisfy this equilibrium equation, the mean delay $\tau_{i}$ may be similar for all large $\alpha_{i}$ of these choices (our numerical simulations also confirm this; see Table 2), and so it might be possible to estimate the mean delay in this manner. The estimate could possibly be improved by repeating the experiment with the same culture but with a different dilution rate or input nutrient concentration.

This also suggests another relatively simple approach for predicting the outcome of competition of two species in a chemostat, without even knowing the form of the response functions or the form of the delay kernels, that could easily be tested by experiments. The theory predicts that the relative values of the $\lambda_{i}$ 's determine the outcome and, as mentioned above, that these $\lambda_{i}$ 's can be determined by culturing each population alone in the chemostat, using the appropriate feed concentration $S^{0}$ and dilution rate $D$, and then just measuring the equilibrium concentration of the nutrient. We refer to Wolkowicz and Xia [48] for a similar discussion on the discrete delay model (1.2).

On a more qualitative level, the numerical simulations (Figure 2) seem to demonstrate that if the death rate, observed in experiments, of the losing population is slower than predicted by the ODEs model, one might suspect that the mean delay in the conversion process of the surviving population is relatively long compared to the 
mean delay of the losing population. On the other hand, if the actual death rate of the losing population is much faster than predicted by the ODEs model, one might suspect that the mean delay in the conversion process of the surviving population is relatively short compared to the mean delay of the losing population. Also, delay may account for more oscillations in the initial transients.

Appendix. In this appendix, we show how the discrete delay model (1.2) and its corresponding ODEs model can be regarded as limiting cases of the distributed delay model (2.1). The results proved here can be extended to general distributed delay models with gamma distribution delay kernels, but for simplicity, we only give the results that are directly related to the present paper.

We begin by considering two nonlinear functionals. Let $\tau>0$ be a constant and $h: \mathbb{R}_{+}^{3} \rightarrow \mathbb{R}$ be a continuous function. We define the following families of functionals on the Banach space $B C_{+}^{3}$ of bounded and continuous functions from $\mathbb{R}_{+}^{3}$ into $\mathbb{R}$ with uniform norm:

$$
\begin{aligned}
& f_{\alpha}(\phi)=\int_{0}^{\infty} h(\phi(-u)) e^{-D u} E_{\alpha}(u) d u, \quad \alpha>0, \\
& g_{r}(\phi)=\int_{0}^{\infty} h(\phi(-u)) e^{-D u} G_{r}(u) d u, \quad r=0,1,2, \ldots,
\end{aligned}
$$

where $\phi \in B C_{+}^{3}, D>0$ is a constant, and

$$
\begin{aligned}
& E_{\alpha}(u)=\frac{\alpha^{p+1} u^{p}}{p !} e^{-\alpha u}, \quad p \geq 0 \text { is a fixed integer, } \\
& G_{r}(u)=\frac{\beta^{r+1} u^{r}}{r !} e^{-\beta u}, \quad \beta=\frac{r+1}{\tau} .
\end{aligned}
$$

Notice that for each $\alpha>0$ and $r=0,1,2, \ldots$,

$$
\int_{0}^{\infty} E_{\alpha}(u) d u=\int_{0}^{\infty} G_{r}(u) d u=1
$$

Thus, functionals (A.1) and (A.2) are well defined. We study the limits of $f_{\alpha}$ and $g_{r}$, as $\alpha \rightarrow \infty$ and $r \rightarrow \infty$, respectively.

ThEOREM A. For any $\phi \in B C_{+}^{3}, \lim _{\alpha \rightarrow \infty} f_{\alpha}(\phi)=h(\phi(0))$.

Proof. Let $\varepsilon>0$ be given. Since $h(\phi(-u)) e^{-D u}$ is continuous at $u=0$, there exists $\delta=\delta(\varepsilon)>0$ such that

$$
\left|h(\phi(-u)) e^{-D u}-h(\phi(0))\right|<\frac{\varepsilon}{2} \text { for all } 0 \leq u \leq \delta .
$$

Let $M=1+\sup _{\theta \in(-\infty, 0]}\left|h(\phi(\theta)) e^{D \theta}-h(\phi(0))\right|$. Since $0<M<\infty$, we can find a number $N=N(\varepsilon)>0$ such that

$$
\int_{\alpha \delta}^{\infty} \frac{v^{p} e^{-v}}{p !} d v<\frac{\varepsilon}{2 M} \text { for all } \alpha \geq N
$$


Therefore, if $\alpha \geq N$,

$$
\begin{aligned}
\left|f_{\alpha}(\phi)-h(\phi(0))\right| & =\left|\int_{0}^{\infty}\left[h(\phi(-u)) e^{-D u}-h(\phi(0))\right] E_{\alpha}(u) d u\right| \\
& \leq\left(\int_{0}^{\delta}+\int_{\delta}^{\infty}\right)\left|h(\phi(-u)) e^{-D u}-h(\phi(0))\right| E_{\alpha}(u) d u \\
& <\frac{\varepsilon}{2} \int_{0}^{\delta} E_{\alpha}(u) d u+M \int_{\delta}^{\infty} E_{\alpha}(u) d u \\
& <\frac{\varepsilon}{2}+M \int_{\delta}^{\infty} \frac{\alpha^{p+1} u^{p} e^{-\alpha u}}{p !} d u \\
& =\frac{\varepsilon}{2}+M \int_{\alpha \delta}^{\infty} \frac{v^{p} e^{-v}}{p !} d v \quad(\text { where } \alpha u=v) \\
& <\frac{\varepsilon}{2}+\frac{\varepsilon}{2}=\varepsilon .
\end{aligned}
$$

This shows that $\lim _{\alpha \rightarrow \infty} f_{\alpha}(\phi)=h(\phi(0))$, as claimed.

THEOREM B. For any $\phi \in B C_{+}^{3}, \lim _{r \rightarrow \infty} g_{r}(\phi)=h(\phi(-\tau)) e^{-D \tau}$.

Proof. Let $\varepsilon>0$ be given. We can find $\delta=\delta(\varepsilon)<\tau$ such that if $|u-\tau| \leq \delta$,

$$
\left|h(\phi(-u)) e^{-D u}-h(\phi(-\tau)) e^{-D \tau}\right|<\frac{\varepsilon}{3} .
$$

This implies that for all $r=0,1,2, \ldots$,

$$
\begin{aligned}
I_{1} & :=\int_{\tau-\delta}^{\tau+\delta}\left|h(\phi(-u)) e^{-D u}-h(\phi(-\tau)) e^{-D \tau}\right| G_{r}(u) d u \\
& <\frac{\varepsilon}{3} \int_{\tau-\delta}^{\tau+\delta} G_{r}(u) d u<\frac{\varepsilon}{3} .
\end{aligned}
$$

Observe that each $G_{r}(u)$ attains a unique maximum value at $u=\frac{r}{\beta}=\frac{r \tau}{r+1}$ and $G_{r}(u)$ is increasing on $\left[0, \frac{r \tau}{r+1}\right]$ and decreasing on $\left[\frac{r \tau}{r+1}, \infty\right)$. If $r \geq R_{1}=\frac{\tau}{\delta}-1$, we have $\frac{r \tau}{r+1} \geq \tau-\delta$ and thus for every $u \in[0, \tau-\delta]$,

$$
\begin{aligned}
G_{r}(u) & \leq G_{r}(\tau-\delta)=\frac{\beta^{r+1}(\tau-\delta)^{r}}{r !} e^{-(\tau-\delta) \beta} \\
& =\frac{(r+1)\left(\frac{r+1}{\tau}\right)^{r+1}(\tau-\delta)^{r}}{(r+1) !} e^{-\frac{\tau-\delta}{\tau}(r+1)} .
\end{aligned}
$$

By Stirling's formula (see Lang [29, Theorem 2, p. 220]), there exists $0<\theta_{r}<1$ such that

$$
(r+1) !=\sqrt{2 \pi(r+1)}\left(\frac{r+1}{e}\right)^{r+1} e^{\frac{\theta_{r}}{12(r+1)}}
$$

and so it follows from (A.4) that for all $u \in[0, \tau-\delta]$,

$$
\begin{aligned}
G_{r}(u) & \leq \frac{e}{\tau \sqrt{2 \pi}}(r+1)^{\frac{1}{2}}\left[\frac{e(\tau-\delta)}{\tau}\right]^{r} e^{-\frac{\tau-\delta}{\tau} r} \\
& =\frac{e}{\tau \sqrt{2 \pi}}(r+1)^{\frac{1}{2}}\left[\frac{a}{e^{a-1}}\right]^{r},
\end{aligned}
$$


where $0<a=\frac{\tau-\delta}{\tau}<1$. Let $M=1+\sup _{\theta \in(-\infty, 0]}\left|h(\phi(\theta)) e^{D \theta}-h(\phi(-\tau)) e^{-D \tau}\right|$. Since $0<M<\infty$ and $a<e^{a-1}$, there exists $R_{2}=R_{2}(\varepsilon) \geq R_{1}$ such that for all $r \geq R_{2}$,

$$
\frac{e}{\tau \sqrt{2 \pi}}(r+1)^{\frac{1}{2}}\left[\frac{a}{e^{a-1}}\right]^{r}<\frac{\varepsilon}{3 M(\tau-\delta)} .
$$

Therefore, by (6), if $r \geq R_{2}$,

$$
G_{r}(u)<\frac{\varepsilon}{3 M(\tau-\delta)} \text { for all } u \in[0, \tau-\delta],
$$

and, as a consequence,

$$
\begin{aligned}
I_{2} & :=\int_{0}^{\tau-\delta}\left|h(\phi(-u)) e^{-D u}-h(\phi(-\tau)) e^{-D \tau}\right| G_{r}(u) d u \\
& <M \int_{0}^{\tau-\delta} G_{r}(u) d u<\frac{\varepsilon}{3} .
\end{aligned}
$$

We now estimate the following integral:

$$
I_{3}:=\int_{\tau+\delta}^{\infty}\left|h(\phi(-u)) e^{-D u}-h(\phi(-\tau)) e^{-D \tau}\right| G_{r}(u) d u .
$$

By using Stirling's formula (A.5) once more, we obtain

$$
\begin{aligned}
I_{3} & \leq M \int_{\tau+\delta}^{\infty} G_{r}(u) d u \\
& \leq \frac{M}{\sqrt{2 \pi}} \int_{\tau+\delta}^{\infty}(r+1)^{\frac{1}{2}}\left(\frac{e}{\tau}\right)^{r+1} u^{r} e^{-\frac{r+1}{\tau} u} d u \\
& =\frac{M}{\tau \sqrt{2 \pi}} \int_{\tau+\delta}^{\infty}(r+1)^{\frac{1}{2}}\left(\frac{u}{\tau} e^{-\frac{u}{\tau}+1}\right)^{r} e^{-\frac{u}{\tau}+1} d u \\
& =\frac{M}{\sqrt{2 \pi}} \int_{1+\frac{\delta}{\tau}}^{\infty}(r+1)^{\frac{1}{2}}\left[v e^{-(v-1)}\right]^{r} e^{-(v-1)} d v,
\end{aligned}
$$

where $v=\frac{u}{\tau}$. Notice that the function $v e^{-(v-1)}$ is decreasing on $[1, \infty)$. Thus, for all $v \geq 1+\frac{\delta}{\tau}$

$$
v e^{-(v-1)} \leq\left(1+\frac{\delta}{\tau}\right) e^{-\frac{\delta}{\tau}}<1
$$

Therefore, there exists $R_{3}=R_{3}(\varepsilon)>0$ such that for all $r \geq R_{3}$,

$$
\frac{M}{\sqrt{2 \pi}}(r+1)^{\frac{1}{2}}\left[v e^{-(v-1)}\right]^{r}<\frac{\varepsilon}{3}, v \in\left[1+\frac{\delta}{\tau}, \infty\right),
$$

and so from (A.8) it follows that if $r \geq R_{3}$,

$$
I_{3} \leq \frac{\varepsilon}{3} \int_{1+\frac{\delta}{\tau}}^{\infty} e^{-(v-1)} d v<\frac{\varepsilon}{3}
$$


Let $R=R(\varepsilon)=\max \left(R_{2}, R_{3}\right)$. It then follows from (A.3), (A.7), and (A.9) that if $r \geq R$

$$
\begin{aligned}
\left|g_{r}(\phi)-h(\phi(-\tau)) e^{-D \tau}\right| & =\left|\int_{0}^{\infty}\left[h(\phi(-u)) e^{-D u}-h(\phi(-\tau)) e^{-D \tau}\right] G_{r}(u) d u\right| \\
& \leq \int_{0}^{\infty}\left|h(\phi(-u)) e^{-D u}-h(\phi(-\tau)) e^{-D \tau}\right| G_{r}(u) d u \\
& =I_{1}+I_{2}+I_{3}<\varepsilon .
\end{aligned}
$$

Therefore, $\lim _{r \rightarrow \infty} g_{r}(\phi)=h(\phi(-\tau)) e^{-D \tau}$.

In what follows, we use Theorems A and B to show that the discrete delay model (1.2) and its corresponding ODEs model are limiting cases of model (2.1). By using the standard notation for functional differential equations (see, for example, Burton [4] and Kuang [28]), we rewrite model (2.1) in the form

$$
x^{\prime}(t)=H_{\boldsymbol{\alpha}, \mathbf{r}}\left(x_{t}\right),
$$

where $x(t)=\left(S(t), x_{1}(t), x_{2}(t)\right) \in \mathbb{R}^{3}, x_{t} \in B C_{+}^{3}$ is defined by $x_{t}(\theta)=x(t+\theta), \theta \in$ $(-\infty, 0], H_{\boldsymbol{\alpha}, \mathbf{r}}: B C_{+}^{3} \rightarrow \mathbb{R}^{3}$ is given by

$$
H_{\boldsymbol{\alpha}, \mathbf{r}}(\phi)=\left(\begin{array}{c}
\left(S^{0}-\phi_{0}(0)\right) D-\phi_{1}(0) p_{1}\left(\phi_{0}(0)\right)-\phi_{2}(0) p_{2}\left(\phi_{0}(0)\right) \\
-D \phi_{1}(0)+\int_{0}^{\infty} \phi_{1}(-u) p_{1}\left(\phi_{0}(-u)\right) e^{-D u} K_{1}(u) d u \\
-D \phi_{2}(0)+\int_{0}^{\infty} \phi_{2}(-u) p_{2}\left(\phi_{0}(-u)\right) e^{-D u} K_{2}(u) d u
\end{array}\right)
$$

$\phi=\left(\phi_{0}, \phi_{1}, \phi_{2}\right) \in B C_{+}^{3}, K_{i}$ are given by $(2.2), \boldsymbol{\alpha}=\left(\alpha_{1}, \alpha_{2}\right)$, and $\mathbf{r}=\left(r_{1}, r_{2}\right)$. Similarly, if we define the functionals

$$
H_{\tau}(\phi)=\left(\begin{array}{c}
\left(S^{0}-\phi_{0}(0)\right) D-\sum_{i=1}^{2} \phi_{i}(0) p_{i}\left(\phi_{0}(0)\right) \\
-D \phi_{1}(0)+e^{-D \tau_{1}} \phi_{1}\left(-\tau_{1}\right) p_{1}\left(\phi_{0}\left(-\tau_{1}\right)\right) \\
-D \phi_{2}(0)+e^{-D \tau_{2}} \phi_{2}\left(-\tau_{2}\right) p_{2}\left(\phi_{0}\left(-\tau_{2}\right)\right)
\end{array}\right)
$$

and

$$
H_{0}(\phi)=\left(\begin{array}{c}
\left(S^{0}-\phi_{0}(0)\right) D-\sum_{i=1}^{2} \phi_{i}(0) p_{i}\left(\phi_{0}(0)\right) \\
-D \phi_{1}(0)+\phi_{1}(0) p_{1}\left(\phi_{0}(0)\right) \\
-D \phi_{2}(0)+\phi_{2}(0) p_{2}\left(\phi_{0}(0)\right)
\end{array}\right)
$$

where $\phi=\left(\phi_{0}, \phi_{1}, \phi_{2}\right) \in B C_{+}^{3}$ and $\boldsymbol{\tau}=\left(\tau_{1}, \tau_{2}\right)$, then model (1.2) can be rewritten as

$$
x^{\prime}(t)=H_{\tau}\left(x_{t}\right)
$$

and the corresponding ODEs model (i.e., model $(1.2)$ with $\tau_{i}=0$ ) takes the form

$$
x^{\prime}(t)=H_{0}\left(x_{t}\right) .
$$

Let $h_{i}\left(x_{0}, x_{1}, x_{2}\right)=x_{i} p_{i}\left(x_{0}\right), i=1,2$. Applying Theorem A, it follows that for any 


$$
\begin{aligned}
& \phi \in B C_{+}^{3}, \\
& \lim _{\substack{\alpha_{1} \rightarrow \infty \\
\alpha_{2} \rightarrow \infty}} H_{\alpha, \mathbf{r}}(\phi)=\left(\begin{array}{c}
\left(S^{0}-\phi_{0}(0)\right) D-\phi_{1}(0) p_{1}\left(\phi_{0}(0)\right)-\phi_{2}(0) p_{2}\left(\phi_{0}(0)\right) \\
-D \phi_{1}(0)+\lim _{\alpha_{1} \rightarrow \infty} \int_{0}^{\infty} \phi_{1}(-u) p_{1}\left(\phi_{0}(-u)\right) e^{-D u} K_{1}(u) d u \\
-D \phi_{2}(0)+\lim _{\alpha_{2} \rightarrow \infty} \int_{0}^{\infty} \phi_{2}(-u) p_{2}\left(\phi_{0}(-u)\right) e^{-D u} K_{2}(u) d u
\end{array}\right) \\
& =\left(\begin{array}{c}
\left(S^{0}-\phi_{0}(0)\right) D-\phi_{1}(0) p_{1}\left(\phi_{0}(0)\right)-\phi_{2}(0) p_{2}\left(\phi_{0}(0)\right) \\
-D \phi_{1}(0)+\lim _{\alpha_{1} \rightarrow \infty} \int_{0}^{\infty} h_{1}(\phi(-u)) e^{-D u} K_{1}(u) d u \\
-D \phi_{2}(0)+\lim _{\alpha_{2} \rightarrow \infty} \int_{0}^{\infty} h_{2}(\phi(-u)) e^{-D u} K_{2}(u) d u
\end{array}\right) \\
& =\left(\begin{array}{c}
\left(S^{0}-\phi_{0}(0)\right) D-\sum_{i=1}^{2} \phi_{i}(0) p_{i}\left(\phi_{0}(0)\right) \\
-D \phi_{1}(0)+h_{1}(\phi(0)) \\
-D \phi_{2}(0)+h_{2}(\phi(0))
\end{array}\right) \\
& =\left(\begin{array}{c}
\left(S^{0}-\phi_{0}(0)\right) D-\sum_{i=1}^{2} \phi_{i}(0) p_{i}\left(\phi_{0}(0)\right) \\
-D \phi_{1}(0)+\phi_{1}(0) p_{1}\left(\phi_{0}(0)\right) \\
-D \phi_{2}(0)+\phi_{2}(0) p_{2}\left(\phi_{0}(0)\right)
\end{array}\right)=H_{0}(\phi),
\end{aligned}
$$

where each $r_{i} \geq 0$ (the order of each $K_{i}$ ) is fixed. Similarly, by letting $\tau=\tau_{i}, i=1,2$, we can apply Theorem B to obtain

$$
\lim _{\substack{r_{1} \rightarrow \infty \\ r_{2} \rightarrow \infty}} H_{\alpha, \mathbf{r}}(\phi)=H_{\tau}(\phi) \text { for any } \phi \in B C_{+}^{3},
$$

where each $\tau_{i}=\frac{r_{i}+1}{\alpha_{i}}$ (the mean delay of each $K_{i}$ ) is fixed. In this sense, we can view systems (A.11) and (A.12) as limiting equations of system (A.10). The discrete delay model (1.2) can be obtained from the distributed delay model (2.1) by letting $r_{i} \rightarrow \infty$ while keeping each mean delay $\tau_{i}=\frac{r_{i}+1}{\alpha_{i}}$ fixed, and the corresponding ODEs model can be obtained from (2.1) by letting each $\alpha_{i} \rightarrow \infty$ while keeping each kernel order $r_{i}$ fixed.

\section{REFERENCES}

[1] J. L. Aron And R. M. May, The population dynamics of malaria, in Population Dynamics of Infectious Diseases: Theory and Applications, R. M. Anderson, ed., Chapman and Hall, London, 1982, pp. 139-179.

[2] N. T. J. BAILey, The Mathematical Theory of Infectious Diseases and Its Applications, Hafner Press, New York, 1975.

[3] E. Beretta AND Y. TAKeuchi, Qualitative properties of chemostat equations with time delay: Boundedness, local and global asymptotic stability, Diff. Eqns. and Dynamical Systems, 2 (1994), pp. 19-40.

[4] T. A. Burton, Volterra Integral and Differential Equations, Academic Press, New York, 1983.

[5] S. N. Busenberg and C. C. Travis, On the use of reducible-functional differential equations in biological models, J. Math. Anal. Appl., 89 (1982), pp. 46-66.

[6] A. W. Bush AND A. E. CoOK, The effect of time delay and growth rate inhibition in the bacterial treatment of wastewater, J. Theoret. Biol., 63 (1975), pp. 385-396.

[7] G. J. Butler And G. S. K. Wolkowicz, A mathematical model of the chemostat with a general class of functions describing nutrient uptake, SIAM J. Appl. Math., 45 (1985), pp. $138-151$.

[8] J. CAPERON, Time lag in population growth response of Isochrysis galbana to a variable nitrate environment, Ecology, 50 (1969), pp. 188-192.

[9] H. A. Caswell, A simulation study of a time lag population model, J. Theoret. Biol., 34 (1972), pp. 419-439.

[10] K. L. Cooke and Z. Grossman, Discrete delay, distributed delay and stability switches, J. Math. Anal. Appl., 86 (1982), pp. 592-627. 
[11] A. Cunningham And R. M. Nisbet, Time lag and co-operativity in the transient growth dynamics of microalgae, J. Theoret. Biol., 84 (1980), pp. 189-203.

[12] J. M. Cushing, Integrodifferential Equations and Delay Models in Population Dynamics, Lecture Notes in Biomathematics 20, Springer-Verlag, Heidelberg, 1977.

[13] K. Dietz, L. Molineaux, And A. Thomas, A malaria model tested in the African savannah, Bull. Wld. Hlth. Org., 50 (1974), pp. 347-357.

[14] M. R. Droop, Vitamin $B_{12}$ and marine ecology. IV. The kinetics of uptake, growth, and inhibition in Monochrysis lutheri, J. Marine Biol. Assoc. UK, 48 (1968), pp. 689-733.

[15] S. F. Ellermeyer, Competition in the chemostat: Global asymptotic behavior of a model with delayed response in growth, SIAM J. Appl. Math., 54 (1994), pp. 456-465.

[16] H. I. Freedman, J. So, And P. Waltman, Coexistence in a model of competition in the chemostat incorporating discrete delay, SIAM J. Appl. Math., 49 (1989), pp. 859-870.

[17] H. I. Freedman, J. So, and P. Waltman, Chemostat competition with time delays, in Biomedical Modelling and Simulation, J. Eisenfeld and D. S. Levine, eds., J. C. Baltzer AG, Amsterdam, 1989, pp. 171-173.

[18] K. Gopalsamy, Stability and Oscillations in Delay Differential Equations of Population Dynamics, Kluwer, Dordrecht, the Netherlands, 1992.

[19] U. HAlBAch, Life table data and population dynamics of the rotifer Brachionus Calyciflorus Pallas as influenced by periodically oscillating temperature, in Effects of Temperature on Ectothermic Organisms, W. Wieser, ed., Springer-Verlag, Heidelberg, 1973, pp. 217-228.

[20] S. R. Hansen and S. P. Hubbell, Single-nutrient microbial competition: Qualitative agreement between experimental and theoretically forecast outcomes, Science, 207 (1980), pp. $1491-1493$.

[21] D. Herbert, R. Elsworth, And R. C. Telling, The continuous culture of bacteria; a theoretical and experimental study, J. Gen. Microbiol., 14 (1956), pp. 601-622.

[22] R. A. Herbert, Low temperature adaptation in bacteria, in Effects of Low Temperature on Biological Membranes, G. J. Morris and A. Clarke, eds., Academic Press, London, 1981, pp. 41-53.

[23] W. M. Hirsch, H. Hanisch, And J. P. Gabriel, Differential equation models of some parasitic infections: methods for the study of asymptotic behavior, Comm. Pure Appl. Math., 38 (1985), pp. 733-753.

[24] S.-B. Hsu, Limiting behavior for competing species, SIAM J. Appl. Math., 34 (1978), pp. $760-763$.

[25] S.-B. Hsu, S. P. Hubbell, And P. Waltman, A mathematical theory for single-nutrient competition in continuous culture of micro-organisms, SIAM J. Appl. Math., 32 (1977), pp. 366-383.

[26] S.-B. Hsu, P. Waltman, And S. F. Ellermeyer, A remark on the global asymptotic stability of a dynamical system modeling two species competition, Hiroshima Math. J., 24 (1994), pp. 435-445.

[27] H. W. Jannasch And R. T. Mateles, Experimental bacterial ecology studied in continuous culture, in Advances in Microbial Physiology, Vol. 11, A. H. Rose and D. W. Tempest, eds., Academic Press, London, 1974, pp. 165-212.

[28] Y. Kuang, Delay Differential Equations with Applications in Population Dynamics, Academic Press, New York, 1993.

[29] S. LANG, A Complete Course in Calculus, Addison-Wesley, Reading, MA, 1968.

[30] B. R. Levin, F. M. StewART, And L. ChAO, Resource-limited growth, competition, and predation: A model and experimental studies with bacteria and bacteriophages, Amer. Natur., 111 (1977), pp. 3-24.

[31] N. MacDonald, Time lag in simple chemostat models, Biotechnol. Bioengrg., 18 (1976), pp. 805-812.

[32] N. MacDonald, Time Lags in Biological Models, Lecture Notes in Biomathematics 27, Springer-Verlag, Heidelberg, 1978.

[33] N. MacDonald, Time delays in chemostat models, in Microbial Population Dynamics, M. J. Bazin, ed., CRC Press, Boca Raton, FL, 1982, pp. 33-53.

[34] N. MacDonald, Biological Delay System: Linear Stability Theory, Cambridge University Press, Cambridge, UK, 1989.

[35] R. M. MAY, Stability and Complexity in Model Ecosystems, Monograph in Population Biology 6, 2nd ed., Princeton University Press, Princeton, NJ, 1974.

[36] R. K. Miller, Nonlinear Volterra Integral Equations, Benjamin, New York, 1971.

[37] J. Monod, La technique de la culture continue: Théorie et applications, Ann. Inst. Pasteur, 79 (1950), pp. 390-401.

[38] S. RuAn And G. Wolkowicz, Bifurcation analysis of a chemostat model with a distributed delay, J. Math. Anal. Appl., 204 (1996), pp. 786-812. 
[39] F. M. Scudo And J. R. Ziegler, The Golden Age of Theoretical Ecology: 1923-1940, Lecture Notes in Biomathematics 22, Springer-Verlag, Berlin, 1978.

[40] H. L. Smith And P. Waltman, The Theory of the Chemostat, Cambridge University Press, Cambridge, UK, 1994

[41] M. L. Stephens And G. Lyberatos, Effect of cycling on final mixed culture fate, Biotechnol. Bioengrg., 29 (1987), pp. 672-678.

[42] C. Strobeck, $N$ species competition, Ecology, 54 (1973), pp. 650-654.

[43] H. R. Thieme, Convergence results and a Poincaré-Bendixson trichotomy for asymptotically autonomous differential equations, J. Math. Biol., 30 (1992), pp. 755-763.

[44] T. F. Thingstad And T. I. Langeland, Dynamics of chemostat culture: The effect of a delay in cell response, J. Theoret. Biol., 48 (1974), pp. 149-159.

[45] D. Tilman, Resource Competition and Community Structure, Princeton University Press, Princeton, NJ, 1982.

[46] H. VeldKamp, Ecological studies with the chemostat, in Advances in Microbial Ecology, Vol. I, M. Alexander, ed., Plenum Press, New York, 1977, pp. 59-94.

[47] G. S. K. Wolkowicz AND Z. Lu, Global dynamics of a mathematical model of competition in the chemostat: general response functions and differential death rates, SIAM J. Appl. Math., 52 (1992), pp. 222-233.

[48] G. Wolkowicz And H. XIA, Global asymptotic behavior of a chemostat model with discrete delays, SIAM J. Appl. Math., 57 (1997), pp. 1019-1043. 